\title{
¿Puede un factor réplica del crecimiento económico futuro (PIB) explicar los rendimientos de los activos financieros cotizados en la bolsa española? *
}

\author{
AlFredo JuAn GraU-GraU \\ Universidad de Valencia, Facultad de Economía, Avda. de los Naranjos, s/n, 46022 Valencia, España. \\ E-mail: alfredo.grau@uv.es
}

\begin{abstract}
RESUMEN
El objetivo de este trabajo es analizar si un factor con capacidad predictiva sobre el crecimiento económico futuro, captura los rendimientos de los activos cotizados en la bolsa española. A su vez, se analiza la posible interpretación racional económica de los factores de Fama y French y momentum, como variables con información sobre dicho crecimiento económico futuro. Se cuantifican estos efectos para las etapas de crisis (económica: 1993-1997 y financiera: 2008-2011) y de expansión económica (1998-2007). Los resultados subrayan la relevancia y capacidad explicativa de este factor predictivo pero se encuentra una interpretación económica débil de los factores tamaño y ratio book-to-market, a partir de este factor que recoge las noticias del mercado sobre el crecimiento futuro del PIB. El factor de negociación momentum aporta un impacto económico prácticamente nulo.
\end{abstract}

Palabras clave: Crecimiento económico futuro, modelos de valoración, explicación racional económica, primas económicas, crisis financiera- 08 y económica-93.

\section{News Related to Future Gross Domestic Product (GDP) Growth Factor on Asset Pricing on the Spanish Stock Market?}

\begin{abstract}
The aim of this paper is to examine whether a factor predictive of future economic growth, captures asset returns on the Spanish stock market. Furthermore, we analyze the possible economic rational interpretation of the Fama and French factors and momentum, as variables with information about the future economic growth. These effects are quantified for the stages of crisis (economic: 1993 to 1997, and financial: 2008 to 2011) and economic expansion (1998 to 2007). The results show the relevance and explanatory power of this predictor in all periods, but is a weak economic interpretation of the size and book-to-market ratio factors, since this factor reflects market news on growth future GDP. The economic impact of momentum factor is irrelevant.

Keywords: Gross Domestic Product, Asset Pricing, Economic Rational Pricing, Economic Premiums, Economic-93 Crisis And Financial-08.

Clasificación JEL: G12

\footnotetext{
* El presente artículo fue presentado en el XX FINANCE FORUM celebrado en Oviedo en noviembre de 2012 a través de la Asociación Española de Finanzas (AEFIN). Además, otra versión previa de este artículo está publicada como documento de trabajo n. ${ }^{\circ} 613 / 2011$ en FUNCAS.

El autor agradece a la Doctora M. ${ }^{a}$ Begoña Font Belaire de la Universidad de Valencia toda la ayuda prestada. Se agradecen también los comentarios de los evaluadores anónimos, los cuales, sin ningún género de dudas, han contribuido a la mejora sustancial del presente artículo.
}

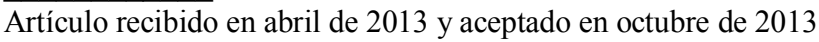

Artículo disponible en versión electrónica en la página www.revista-eea.net, ref. ə-32201 


\section{INTRODUCCIÓN}

El "clásico" modelo de tres factores ${ }^{1}$ de Fama y French (1993), hasta nuestros días, sigue siendo un modelo de valoración de referencia para todo inversor que pretenda cuantificar adecuadamente los rendimientos de sus carteras. No obstante, la relevancia de este modelo ha generado todo tipo de controversias, siendo la más destacada la interpretación de los factores que integra (tamaño, ratio book-to-market y, en su caso, momentum) como factores de riesgo sistemático. Fama y French (1995) defienden una interpretación racional de los factores tamaño y ratio book-to-market basada en la capacidad de estos efectos para explicar el comportamiento de los resultados de las empresas. Adicionalmente, Fama y French $(1993,1996)$ también argumentan que estos factores actúan como variables de estado en el contexto del modelo CAPM intertemporal de Merton (1973). Al hilo de la segunda línea de investigación, Liew y Vassalou (2000) muestran que los factores tamaño y ratio book-to-market contienen información predictiva sobre el crecimiento económico pero obtienen poca evidencia para soportar esa misma hipótesis para un factor momentum a un año. Font y Grau (2007) realizan este análisis con datos españoles y concluyen que los factores tamaño, ratio book-to-market y momentum a 1, 3 y 6 meses contribuyen conjunta y significativamente en la predicción del crecimiento de las variables macroeconómicas producto interior bruto (PIB), renta y salarios. Y Vassalou (2003), da el paso incremental al proponer un modelo de valoración con factor mercado y un nuevo factor de riesgo (en nuestro artículo factor réplica del PIB) que recoge las noticias relativas al crecimiento futuro de PIB y aportan evidencias empíricas que indican que la capacidad de este modelo, para explicar los rendimientos en sección cruzada, es similar a la del modelo de tres factores de Fama y French (1993), y que al sobredimensionar este modelo incluyendo estos factores pierden gran parte de su capacidad para explicar los rendimientos.

El objetivo de este artículo consiste, en primer lugar, cuantificar la contribución de un factor réplica con capacidad predictiva sobre el crecimiento económico futuro, en los rendimientos de los activos en sección cruzada considerando tres subperíodos que registran la crisis económica de 1993 y financiera del 2008 (1993-1997 y 2008-2011), y otra de expansión económica (1998-2007); así como el período completo. Y, en segundo lugar, estudiar si los factores tamaño,

\footnotetext{
${ }^{1}$ Entre los autores que han estimado y contrastado este modelo, en alguna de sus variantes, en el mercado de capitales español se pueden citar: Nieto y Rodríguez (2005) empleando rendimientos trimestrales desde marzo 1982 a diciembre 1999, Nieto (2004) usando rendimientos mensuales para el periodo 1982-1998, Miralles y Miralles $(2003,2007)$ utilizando rendimientos mensuales para el periodo 1998-2002, y Font y Grau (2007) usando rendimientos diarios para el periodo 1995-2000.
} 
ratio book-to-market y momentum ${ }^{2}$ admiten una explicación racional económica, como variables de estado con información sobre el crecimiento económico futuro. Se plantean estos objetivos a raíz de realizar un minucioso proceso de revisión de la literatura financiera para el mercado de capitales español. Se detecta una ausencia de trabajos previos que estudien, por un lado, la influencia del factor réplica del PIB, y por otro verificar si los clásicos factores de Fama y French y momentum, pueden admitir una explicación racional de tipo económico. Es más, no existen artículos que desarrollen estos contenidos en el proceso de valoración de los activos financieros.

Este artículo se inspira, en parte, en los trabajos de Vassalou (2003) y Lamont (2001) en cuanto al procedimiento de construcción de los factores. Es importante notar que en el presente trabajo se analiza el efecto réplica del PIB a través de la estimación y contrastación de modelos de valoración, y su impacto económico de las primas económicas. A nuestro entender, se trata de una cuestión fundamental para profundizar y llegar a formular argumentos y conclusiones relevantes, de cuyo estudio y desarrollo carece el mercado de capitales español, en general, y los trabajos de Vassalou, en particular.

La contribución empírica de este artículo es abundante: (i) la cuantificación de los factores en cada uno de los modelos, en términos de primas y del impacto económico (premia) para explicar los rendimientos de sección cruzada. El estudio separa la muestra completa en tres subperiodos que permite observar este comportamiento en función de dos fases de crisis y una fase de expansión económica; (ii) la cuantificación de la contribución en los rendimientos del factor réplica (explicación racional económica) atendiendo a los tres subperíodos; y (iii) la cuantificación de los factores tamaño, ratio book-to-market y momentum descontado el factor réplica en la explicación de los rendimientos, y de este modo, estudiar la validez de las explicaciones racionales analizadas.

Los resultados obtenidos se pueden resumir de la siguiente manera: (i) el modelo de tres factores de Fama y French (1993) es el modelo que mejor describe los rendimientos de sección cruzada de activos españoles para las dos agrupaciones de activos consideradas; (ii) los resultados basados en el impacto económico de los factores muestran una escasa interpretación de los factores tamaño y ratio book-to-market como riesgos asociados al factor réplica del PIB; y (iii) ninguna de las explicaciones racionales estudiadas permite interpretar como factor de riesgo una variable momentum a 3, 6, 9 y 12 que además tiene un impacto económico prácticamente inexistente.

El resto del artículo se organiza de la siguiente manera. En la Sección 2 se presentan los datos y se explica la construcción de las carteras y de los factores.

\footnotetext{
${ }^{2}$ La inclusión del efecto momentum en el análisis de esta explicación se apoya en las evidencias empíricas que presentan Font y Grau (2007) para el mercado de capitales español.
} 
En la Sección 3 se describen los modelos de valoración empleados en el estudio y se expone la metodología aplicada. En la Sección 4 se detallan los resultados empíricos del artículo: la contribución de los factores de los modelos en términos de primas al riesgo y primas económicas y la naturaleza racional de los factores tamaño y ratio book-to-market y efecto momentum a partir de las dos explicaciones racionales propuestas. Y en la Sección 5 se termina con un resumen de los resultados y conclusiones más importantes.

\section{DATOS Y CARTERAS}

Los resultados de este estudio se basan en los rendimientos totales mensuales ajustados por dividendos de acciones individuales cotizadas en el mercado continuo español agrupados en dos categorías de carteras: por sector y clasificadas por tamaño y ratio book-to-market (tamaño-BM) para el periodo comprendido entre enero de 1993 y diciembre de 2011.

Estudiamos la muestra completa y una escisión de la misma en tres subperíodos: crisis económica del 1993 (1993-1997), fase de crecimiento económico (1998-2007), y crisis financiera de 2008 (2008-2011). En las últimas décadas la economía española ha pasado por periodos bastante heterogéneos (véase EEAG, 2011). Desde etapas de crisis más o menos duras, a una etapa de bonanza económica. Ello nos conduce a pensar que subdividir la muestra completa en tres grandes subperiodos, ayudan a caracterizar mucho más nuestros datos y a identificar matices que nos ayuden a alcanzar resultados mucho más precisos. El periodo muestral comienza con la crisis económica de 1993, caracterizada por las distintas devaluaciones de la moneda por parte del Gobierno español, España encabeza la tasa de paro de los países de la CEE, el déficit público llega a ser muy alarmante, etc. En 1995 comienzan a verse leves síntomas de mejoría pero no resultan ser notables hasta la entrada en vigor del Pacto de Toledo en 1997. El final de la etapa anterior da paso a una nueva etapa de prosperidad económica (1998). A partir de este instante, la economía española crece por encima de la media europea, se genera mucho empleo, etc. Pero el fuerte crecimiento realmente es debido al sector de la construcción que fue un motor importante para la economía española. Hubo un sustancial aumento de la inversión en bienes de capital, así como también de la inversión en el sector público. No obstante, a partir del 2007 no cesan las noticias que señalan que el sector financiero estadounidense está asumiendo excesivos riesgos con las hipotecas "subprime" cuyo volumen comienza a ser muy preocupante. Con el anuncio de la Bancarrota de Lehman Brothers (el cuarto grupo financiero más importante de EE.UU.) en septiembre de 2008, se inicia la crisis financiera actual, finalizando este estudio a finales del 2011.

En este apartado se describen también los datos y las carteras, los factores de riesgo y las variables instrumentales. 


\subsection{Datos}

Los rendimientos mensuales totales netos de los activos individuales se obtienen a partir de las series de precios diarios a cierre corregidos por ampliaciones y reducciones de capital facilitados por INTERTELL, añadiendo los datos de las empresas que cotizaron durante el periodo de estudio. Las que fueron excluidas del mercado se eliminaron en la actualización de la base. Para obtener estos rendimientos se usaron las cotizaciones del último día del mes corregidas por dividendos netos (dividendo anual bruto corregido por impuestos, usando la corrección propuesta por STOXX para España, distribuido uniformemente entre los 12 meses). La base después de eliminar aquellos activos sin información sobre dividendos está formada por 123 activos.

Los restantes datos para la estimación de los modelos son: las series del índice Ibex-35 e Ibex-35 con dividendos proporcionadas por la SOCIEDAD DE BOLSAS, para la obtención de los rendimientos mensuales de la cartera de mercado; y el tipo medio de operaciones con pacto de recompra a 1 año en letras y bonos facilitado por el BANCO DE ESPAÑA, para calcular los excesos de los rendimientos respecto a la tasa libre de riesgo. La variable instrumental ratio dividendo-precio (div) se calcula a partir de las series del índice Ibex-35 e Ibex-35 con dividendos, dividido por el precio cotizado; y la variable instrumental diferencial de tipos (term) a partir de los tipos medios de letras y bonos a 1 y de bonos a 4-5 años facilitados por el BANCO DE ESPAÑA. Por último, los datos económicos para la construcción de la cartera réplica del mercado sobre el crecimiento futuro del PIB proceden de EUROSTAT; y los datos contables para obtener las carteras clasificadas por tamaño y ratio book-to-market y los factores de riesgo tamaño y ratio book-to-market y efectos momentum se obtienen de SABI e INTERTELL y complementariamente a través de la información contable facilitada por las BOLSAS DE MADRID, BARCELONA Y VALENCIA.

\subsection{Elaboración de las carteras y factores de Fama y Frech más momentum}

La agrupación sectorial contiene 6 carteras correspondientes a seis sectores (energía, industria, bienes, servicios, financiero y tecnológico). Los activos se asignan a cada una de las carteras según esta naturaleza y se ponderan por capitalización, de modo que el peso asignado a cada activo se actualiza mensualmente y es igual al cociente del promedio para el mes anterior de la capitalización de cada activo sobre el total de promedios mensuales del sector al que pertenece. La otra agrupación, tamaño-BM, está formada por 9 carteras ordenadas de menor a mayor tamaño y ratio book-to-market, adaptando nuestro procedimiento al propuesto en Fama y French (1993). La composición de las carteras se actualiza mensualmente con los datos provenientes del mes anterior ordenando los activos de menor a mayor promedio de capitalización y paralelamente de menor a mayor promedio ratio book-to-market. Seguidamente, se 
forman tres clases de (aproximadamente) igual número de activos, esto es, baja $(\mathrm{L})$, media (M) y alta (H); siendo el peso de cada activo en su cartera proporcional al número de activos que forma parte de la misma. Moskowiz y Grinblatt (1999) encuentran un componente específico momentum a nivel industria y ello hace pensar que ciertos efectos pueden ser característicos de algunos sectores, de ahí la justificación de la primera agrupación; captando así, el diferente impacto de los distintos efectos que se pueden observar por la pertenencia a un determinado sector. Por otro lado, la segunda agrupación, atendiendo a las evidencias vertidas, entre otros, por Conrad, Gultekin y Kaul (1991) que argumentan que se puede predecir la volatilidad condicional asimétrica que se pueden dar entre empresas grandes y empresas pequeñas, manifestando así la relevancia del volumen de capitalización de las mismas.

La Tabla 1 (Paneles A y B) muestra los estadísticos descriptivos para las dos agrupaciones de carteras para período completo y tres subperíodos. Destaca: (i) la escasez de estructuras dinámicas en medias y varianzas tanto para toda la extensión de la muestra como para la etapa de bonanza económica; para los períodos de crisis se observa una presencia ligeramente mayor de estas dinámicas y (ii) los excesos de los rendimientos $\left(\mathrm{H}_{0}\right.$ : $\left.\mathrm{ER}=0\right)$ son significativos y positivos para todas las carteras por sector y periodos; en cuanto a la agrupación por tamaño-BM, las únicas carteras significativas son $\mathrm{HH}, \mathrm{MH}$ y MM para las fases de crisis económica-93 y expansión económica, respectivamente. En todos los casos no se observan rendimientos negativos para ninguna cartera.

Se aprecia para el periodo completo que le patrón de comportamiento es muy similar. La media de los rendimientos para las dos agrupaciones es siempre positiva, aunque los niveles más bajos se sitúan en el período de crisis financiera- 08 , como era de esperar.

Junto con estas carteras se calculan los factores de riesgo mercado (EXM), tamaño (SMB), ratio book-to-market (HML) y el factor momentum a 3, 6, 9 y 12 meses (MOM_3, MOM_6, MOM_9 y MOM_12) ${ }^{3}$, y las variables instrumentales ratio dividendo-precio (div) y diferencial de tipos (term). La utilización de estas variables medidoras de la evolución del ciclo económico (instrumentales) está basada en estudios empíricos españoles. Los trabajos más destacados que utilizan un diferencial de rendimientos de letras del tesoro de dos períodos pertenecen a Muga y Santamaría (2007), Nieto (2002) y Rubio (1991). Trabajos que han utilizado el ratio dividendo-precio encontramos a Muga y Santamaría (2007), Nieto (2002, 2004), Nieto y Rodríguez (2002) y Nieto y Rubio (2002). El agregado del ratio book-to-market se implementan en

\footnotetext{
${ }^{3}$ Encontramos antecedentes empíricos en el trabajo de Font y Grau (2009) en la utilización de estos cuatro factores de negociación momentum.
} 
los trabajos de Muga y Santamaría (2007), Nieto (2002, 2004), Nieto y Rodríguez $(2002,2005)$ y Nieto y Rubio (2002).

Tabla 1

Estadísticos descriptivos para las carteras por Sector y Tamaño-BM y cartera réplica $R \triangle P I B$

Panel A: Estadísticos descriptivos para la agrupación por Sector

\section{Panel A.0: período completo (93-11)}

\begin{tabular}{|c|c|c|c|c|c|c|}
\hline Sector & Media & DS & JB & $Q(6)$ & $Q^{2}(6)$ & $H_{0}: E R=0$ \\
\hline ENERGIA & 0.03839 & 0.03534 & $94.743^{* *}$ & 8.8351 & 9.8516 & $3.2677^{\star *}$ \\
\hline INDUST. & 0.07307 & 0.02813 & $41.125^{\star *}$ & $10.825^{*}$ & $19.861^{*}$ & $7.1189^{* *}$ \\
\hline BIENES & 0.01821 & 0.07419 & $78.259^{* *}$ & 6.3084 & $16.973^{\star}$ & $4.1367^{\star}$ \\
\hline SERVIC. & 0.02412 & 0.09109 & $409.54^{\star *}$ & 4.0062 & 9.6741 & $3.0098^{*}$ \\
\hline FINANC. & 0.01318 & 0.05174 & $31.146^{* *}$ & 7.1678 & $21.933^{\star}$ & $5.1877^{\star *}$ \\
\hline TECNOL. & 0.02035 & 0.10936 & $9.17 .55^{\star \star}$ & $12.961^{*}$ & $26.971^{*}$ & $8.128^{* *}$ \\
\hline \multicolumn{7}{|c|}{ Panel A.2: período expansión (98-07) } \\
\hline Sector & Media & DS & JB & $Q(6)$ & $Q^{2}(6)$ & $H_{0}: E R=0$ \\
\hline ENERGIA & 0.06347 & 0.01673 & $53.533^{* *}$ & 5.3621 & 5.9745 & $4.8741^{* *}$ \\
\hline INDUST. & 0.07851 & 0.02411 & $19.006^{* *}$ & 7.0567 & $11.790^{*}$ & $5.0089^{* *}$ \\
\hline BIENES & 0.02108 & 0.06107 & $93.507^{* *}$ & 6.7621 & 9.0051 & $3.1047^{\star}$ \\
\hline SERVIC. & 0.02934 & 0.05342 & $208.37^{\star \star *}$ & 3.0584 & 4.0452 & $2.9823^{\star}$ \\
\hline FINANC. & 0.01432 & 0.02774 & $15.615^{\star *}$ & 8.6692 & $9.087^{*}$ & $4.3601^{* *}$ \\
\hline TECNOL. & 0.02509 & 0.09636 & $6.4731^{* *}$ & $11.006^{*}$ & $15.762^{* \star}$ & $9.5471^{* *}$ \\
\hline
\end{tabular}

Panel B: Estadísticos descriptivos para la agrupación Tamaño-BM Panel B.0: período completo (93-11)

\begin{tabular}{lcccccc}
\hline Tam-book & Media & DS & JB & $Q(6)$ & $Q^{2}(6)$ & $\boldsymbol{H}_{0}: E R=0$ \\
\hline LL & 0.01153 & 0.12140 & $371.969^{* *}$ & 2.95989 & 5.95635 & 1.4973 \\
LM & 0.00860 & 0.07033 & $51.2655^{\star *}$ & $3.5441^{\wedge}$ & $10.834^{\star}$ & 1.2172 \\
LH & 0.01359 & 0.07776 & $398.269^{* *}$ & 5.34127 & 10.8045 & $4.9232^{\wedge}$ \\
ML & 0.00801 & 0.06311 & $285.295^{\star *}$ & 3.14653 & $13.2449^{\star}$ & 2.0387 \\
MM & 0.01575 & 0.04221 & $36.1048^{\star *}$ & $13.322^{\wedge}$ & $17.2411^{\star}$ & $3.9201^{\star}$ \\
MH & 0.01563 & 0.11835 & $15.3149^{\star *}$ & 9.97026 & $38.317^{\star *}$ & $5.2585^{\star}$ \\
HL & 0.01069 & 0.10151 & $2714.46^{\star *}$ & 5.08129 & 3.0908 & 1.2142 \\
HM & 0.01497 & 0.05163 & $19.2541^{\star *}$ & 4.70644 & 5.5909 & $6.3374^{\wedge}$ \\
HH & 0.01757 & 0.06793 & 2.7615 & 1.87709 & 6.7505 & $2.7780^{\star}$ \\
\hline
\end{tabular}

Panel B.2: período expansión (98-07)

\begin{tabular}{|c|c|c|c|c|c|c|}
\hline Tam-book & Media & $D S$ & $J B$ & $Q(6)$ & $Q^{2}(6)$ & $H_{0}: E R=0$ \\
\hline $\mathrm{LL}$ & 0.01315 & 0.07624 & $295.812^{* *}$ & 2.3045 & 6.4679 & 1.0323 \\
\hline LM & 0.01242 & 0.11995 & $39.4027^{* *}$ & 1.6379 & 3.1296 & 0.9672 \\
\hline LH & 0.01533 & 0.05961 & $312.132^{* *}$ & 2.3155 & 5.9393 & $7.8704^{\wedge}$ \\
\hline$M L$ & 0.00757 & 0.07151 & $40.5406^{\star *}$ & 2.0091 & $11.033^{* *}$ & 0.2694 \\
\hline MM & 0.01730 & 0.03994 & $29.6542^{\star *}$ & 9.9634 & $12.329^{\star}$ & $2.4378^{*}$ \\
\hline $\mathrm{MH}$ & 0.01636 & 0.03770 & $9.1447^{\star \star}$ & 8.057 & $22.835^{\star *}$ & $2.2863^{\star}$ \\
\hline $\mathrm{HL}$ & 0.01214 & 0.11617 & $1961.52^{* *}$ & 3.9533 & 0.6419 & 0.917 \\
\hline $\mathrm{HM}$ & 0.01410 & 0.05016 & $9.0238^{\star \star}$ & 3.1008 & 2.9197 & $8.8477^{\wedge}$ \\
\hline $\mathrm{HH}$ & 0.02059 & 0.06975 & 2.45214 & 1.2669 & 9.1955 & $2.5001^{*}$ \\
\hline
\end{tabular}

Panel A.1: período crisis económica (93-97)

\begin{tabular}{|c|c|c|c|c|c|}
\hline Media & DS & JB & $Q(6)$ & $Q^{2}(6)$ & $H_{0}: E R=0$ \\
\hline 0.00855 & 0.08156 & $63.754^{\star *}$ & 5.8489 & 4.5382 & $2.9043^{* \star}$ \\
\hline 0.07157 & 0.09965 & $21.076^{\star \star}$ & 6.6558 & $18.583^{\star \star}$ & $2.5124^{*}$ \\
\hline 0.01826 & 0.06357 & $51.411^{\star *}$ & 7.1117 & 8.4977 & $2.4893^{*}$ \\
\hline 0.00976 & 0.10062 & $196.72^{\star *}$ & 2.4254 & 1.462 & $2.6016^{*}$ \\
\hline 0.01063 & 0.05834 & $9.5141^{*}$ & 7.6816 & $23.107^{\star \star}$ & $3.1926^{\star \star}$ \\
\hline 0.01911 & 0.09644 & $8.9516^{*}$ & 9.6191 & $16.571^{\star \star}$ & $2.2419^{*}$ \\
\hline \multicolumn{6}{|c|}{ Panel A.3: período crisis financiera (08-11) } \\
\hline Media & DS & JB & $Q(6)$ & $Q^{2}(6)$ & $\mathrm{H}_{0}: \mathrm{ER}=0$ \\
\hline 0.00954 & 0.15743 & $71.712^{\star *}$ & 7.4871 & 5.5487 & $1.6478^{*}$ \\
\hline 0.04871 & 0.24734 & $22.095^{\star *}$ & $9.481^{* *}$ & $21.547^{\star \star}$ & $2.4714^{*}$ \\
\hline 0.01005 & 0.09841 & $74.252^{\star *}$ & 4.1578 & $9.4157^{\star}$ & $1.8105^{*}$ \\
\hline 0.00772 & 0.16176 & $394.15^{* *}$ & 2.4478 & 2.231 & $2.83104^{*}$ \\
\hline 0.00974 & 0.09333 & $18.187^{\star *}$ & $8.2101^{\wedge}$ & $29.178^{\star \star}$ & $1.8421^{*}$ \\
\hline 0.01745 & 0.11497 & $11.745^{\star *}$ & 10.602 & $20.513^{* *}$ & $2.0041^{*}$ \\
\hline
\end{tabular}

Panel B.1: período crisis económica (93-97)

\begin{tabular}{|c|c|c|c|c|c|}
\hline Media & $D S$ & $J B$ & $Q(6)$ & $Q^{2}(6)$ & $H_{0}: E R=0$ \\
\hline 0.00710 & 0.10165 & $308.853^{* *}$ & 2.9466 & 7.4112 & 3.1573 \\
\hline 0.00360 & 0.07991 & $46.4004^{* *}$ & 3.3259 & $8.052^{* *}$ & 1.5072 \\
\hline 0.00870 & 0.07120 & $395.132^{* *}$ & 7.3428 & 7.6793 & $2.8457^{\wedge}$ \\
\hline 0.00110 & 0.08543 & $43.5476^{\star \star}$ & 2.7003 & $17.068^{* *}$ & 3.4044 \\
\hline 0.00930 & 0.06092 & $36.0315^{\star \star}$ & $11.953^{\wedge}$ & $13.329^{*}$ & $3.4888^{*}$ \\
\hline 0.00940 & 0.08794 & $10.1445^{\star \star}$ & 10.613 & $44.832^{* \star}$ & $4.1673^{*}$ \\
\hline 0.00640 & 0.10276 & $2632.82^{* *}$ & 5.9548 & 2.3519 & 1.9444 \\
\hline 0.00820 & 0.02197 & $12.0288^{* *}$ & 3.4886 & 3.8197 & $7.9997^{\wedge}$ \\
\hline 0.01120 & 0.06860 & 1.44714 & 2.2614 & 3.1955 & $3.5441^{*}$ \\
\hline \multicolumn{6}{|c|}{ Panel B.3: período crisis financiera (08-11) } \\
\hline Media & DS & $J B$ & $Q(6)$ & $Q^{2}(6)$ & $H_{0}: E R=0$ \\
\hline 0.00890 & 0.15754 & $405.731^{\star \star}$ & 3.0458 & 3.1004 & 0.8741 \\
\hline 0.00420 & 0.21140 & $55.7102^{* *}$ & $5.1497^{*}$ & $19.147^{\star \star}$ & 1.0804 \\
\hline 0.01050 & 0.09435 & $407.941^{* *}$ & 6.7874 & 15.7487 & 1.0472 \\
\hline 0.01010 & 0.04577 & $640.789^{* *}$ & 4.0048 & $10.787^{\star}$ & 3.0578 \\
\hline 0.01310 & 0.03021 & $35.1786^{* *}$ & $14.874^{*}$ & $21.027^{\star \star}$ & $5.0087^{*}$ \\
\hline 0.01400 & 0.20486 & $21.7841^{\star \star}$ & 9.5479 & $44.832^{\star \star}$ & $8.2046^{*}$ \\
\hline 0.00840 & 0.18484 & $3017.84^{* *}$ & 4.7842 & 5.74875 & 0.917 \\
\hline 0.01570 & 0.09052 & $30.8410^{\star *}$ & 6.1458 & 8.41771 & 0.78411 \\
\hline 0.01270 & 0.10340 & 3.11494 & 1.9784 & 3.78402 & $2.0478^{*}$ \\
\hline
\end{tabular}


Tabla 1(continuación)

Estadísticos descriptivos para las carteras por Sector y Tamaño-BM y cartera réplica $R \triangle P I B$

Panel C: Cartera réplica RAPIB

Panel C.0: período completo (93-11)

\begin{tabular}{lccc}
\hline & Activos Base & \multicolumn{2}{c}{ Variables de control } \\
\hline c & -0.00906 & $\operatorname{EXM}(-1)$ & $-0.02037^{*}$ \\
$\mathrm{HHH}$ & $0.13592^{\star *}$ & $\mathrm{SMB}(-1)$ & -0.04211 \\
$\mathrm{HHL}$ & -0.03988 & $\mathrm{HML}(-1)$ & -0.13430 \\
$\mathrm{HLH}$ & $-0.18405^{\star *}$ & $\operatorname{div}(-1)$ & $0.09820^{* *}$ \\
$\mathrm{HLL}$ & $0.04048^{* *}$ & $\operatorname{term}(-1)$ & $1.67382^{*}$ \\
LHH & 0.02091 & & \\
LHL & -0.01803 & & \\
LLH & 0.08697 & & \\
LLL & 0.00608 & & \\
term & 0.86150 & & \\
def & 0.54962 & & \\
\hline
\end{tabular}

Panel C.2: período expansión (98-07)

\begin{tabular}{lccc}
\hline & \multicolumn{2}{c}{ Activos Base } & \multicolumn{2}{c}{ Variables de control } \\
\hline c & -0.002971 & EXM(-1) & $-0.02451^{*}$ \\
HHH & $0.12765^{\star *}$ & SMB $(-1)$ & -0.00967 \\
HHL & -0.030943 & HML $(-1)$ & -0.08726 \\
HLH & $-0.19372^{\star \star}$ & $\operatorname{div}(-1)$ & $0.08034^{\star *}$ \\
HLL & $0.01527^{* *}$ & $\operatorname{term}(-1)$ & 0.48634
\end{tabular}

LHH $\quad 0.037621$

LHL $\quad-0.029462$

LLH $\quad 0.053074$

LLL $\quad 0.006539$

term $\quad 0.663546$

def $\quad 0.364418$

Panel D: Estadísticos descriptivos del factor RAPIB

Panel D.0: período completo (93-11)

\begin{tabular}{ccccc}
\hline & Media & DS & J-B & Ho $_{0}:$ ER $=\mathbf{0}$ \\
\hline R $\triangle$ PIB & 0.000649 & 0.02176 & 4.048716 & 0.87654 \\
\hline
\end{tabular}

Panel D.2: período Expansión (98-07)

\begin{tabular}{ccccc}
\hline & Media & DS & J-B & H $_{0}:$ ER $=\mathbf{0}$ \\
\hline R $\triangle$ PIB & 0.000851 & 0.009075 & 2.006741 & 0.59841
\end{tabular}

La Tabla presenta en los Paneles $A$ y $B$ los siguientes estadísticos descriptivos para las carteras sectoriales $y$ tamaño-BM separando el período completo de los subperíodos de crisis económica y financiera, y de expansión económica: el valor medio de los rendimientos, su desviación estándar (DS) y el test de Jarque-Bera (Ho: Normalidad). Se proporcionan el contraste $Q$ de Ljung-Box para 6 retardos para el estudio de las dinámicas en media $(Q(6))$ y para estudiar las dinámicas en varianzas $\left(Q^{2}(6)\right)$. En los Paneles $C$ y $D$, se proporcionan los resultados de la regresión para la obtención de la cartera réplica $R \triangle P I B$ y los estadísticos descriptivos para $R \Delta P I B$, respectivamente. Finalmente, y para todos los casos, se facilita el contraste de media igual a cero para los excesos de los rendimientos $\left(H_{0}: E R=0\right)$.

Nivel de significatividad de los contrastes: $10 \%\left({ }^{\wedge}\right), 5 \%\left(^{*}\right)$ y $1 \%\left(^{* *}\right)$

Fuente: Elaboración propia.

El factor EXM se obtiene restando a los rendimientos mensuales totales de la cartera de mercado (índice Ibex-35) el activo libre de riesgo (tipo medio de operaciones con pacto de recompra a 1 año en letras y bonos). Los factores SMB y HML se obtienen a partir de las carteras tamaño-BM mediante las ecuaciones: $\mathrm{SMB}=(\mathrm{LL}+\mathrm{LM}+\mathrm{LH}) / 3-(\mathrm{HL}+\mathrm{HM}+\mathrm{HH}) / 3 \quad$ y $\quad \mathrm{HML}=(\mathrm{LH}+\mathrm{MH}+\mathrm{HH}) / 3$ 
$(\mathrm{LL}+\mathrm{ML}+\mathrm{HL}) / 3$. Para cuantificar el efecto sobre el rendimiento de las acciones españolas de los efectos momentum (o contrarian) ${ }^{4}$ se consideran cuatro efectos momentum a 3, 6, 9 y 12 meses (MOM_3, MOM_6, MOM_9 y MOM_12) que se obtienen clasificando las empresas, a partir de los rendimientos totales netos 3, 6, 9 y 12 meses $^{5}$ antes de la correspondiente fecha del factor momentum, en ganadoras, intermedias y perdedoras; y calculando este factor a partir de la diferencia de los promedios de los rendimientos de las carteras ganadoras menos las carteras perdedoras. Finalmente, para introducir en los modelos de valoración la información condicional sobre el ciclo económico se definen dos variables instrumentales $^{6}$ : el ratio dividendo-precio (div) y el diferencial tipos a corto y medio/largo plazo (term). La variable instrumental div se calcula a partir de las series del índice Ibex-35 e Ibex-35 con dividendos, corrigiendo los dividendos brutos por impuestos y dividiendo por el precio cotizado, y la variable instrumental term restando los tipos medios de bonos de 4-5 años a los tipos de letras y bonos de 1 año.

\subsection{Factor réplica del crecimiento futuro del PIB}

Principalmente, el trabajo de Vassalou (2003) analiza si el crecimiento del PIB contiene información relevante sobre los rendimientos de los activos financieros. Para profundizar en su cometido construye una cartera réplica que permita al inversor predecir el crecimiento futuro del PIB. Esta cartera réplica se sustenta en el modelo ICAPM cuyas fuentes de riesgo son el mercado y otro factor de riesgo indicativo de las futuras oportunidades de inversión. Por tanto, esa es la razón por la que se estima esta cartera réplica en lugar de utilizar directamente el crecimiento del $\mathrm{PIB}^{7}$. Siguiendo esta línea de trabajo, en este artículo se construye el factor réplica (R $\triangle \mathrm{PIB})$ para llevar al campo empírico estas pretensiones, adaptando el procedimiento propuesto en Vassalou (2003) y La-

\footnotetext{
${ }^{4}$ Forner y Marhuenda (2003) usando rendimientos mensuales ajustados documentan, para el mercado de capitales español y periodo comprendido entre enero de 1963 y diciembre de 1997, evidencias significativas de efectos momentum a 6 y 12 meses y contrarian a 36 y 60 meses. Además, observan que estos fenómenos momentum no pueden ser explicados basándose en un ajuste por riesgo tomando como referencia el modelo CAPM.

${ }^{5}$ Para no perder datos iniciales del factor para el año 1993, se usaron los rendimientos de 1992 , sin corregir dividendos, para obtener las clasificaciones iniciales de las empresas en ganadoras, intermedias y perdedoras.

${ }^{6}$ La elección de estas variables forman parte de las más empleadas en la literatura internacional de valoración condicional de activos; véase, p.e, Fama y French $(1988,1989)$, Cohrane (1996) y Ferson y Harvey $(1991,1999)$.

${ }^{7}$ Duarte, Venetis y Payá (2004) proponen un modelo de regresión lineal basado en el crecimiento del PIB, aunque la metodología y los objetivos que se plantea en su trabajo son bien distintos a los planteados en el presente trabajo.
} 
mont (2001), repectivamente. Los pasos para obtener la cartera réplica, son los siguientes:

Paso 1: Obtención de la serie crecimiento trimestral del PIB ( $\triangle \mathrm{PIB})$. Se calcula diferenciando la serie cuatrimestral del PIB $\left(\Delta \mathrm{PIB}_{\mathrm{t}, \mathrm{t}+3}=\mathrm{PIB}_{\mathrm{t}+3}-\mathrm{PIB}_{\mathrm{t}}\right)$ a precios constantes de 1996 que se obtiene transformando la serie "Quarterly gross domestic product in current prices seasonally adjusted" a precios constantes de 1996. La transformación a precios constantes se realiza a partir de las series mensuales "Various consumption goods and services" para el periodo anterior a 1996 a partir de dicha fecha. (Fuente: EUROSTAT.)

Paso 2: Selección de los activos base y variables de control. Para obtener la cartera réplica se usan 10 activos base, representativos de la información que proporciona el mercado de renta variable y fija, y 5 variables control con capacidad para recoger información sobre el ciclo económico, que seguidamente se procede a explicar:

En primer lugar, 8 carteras agrupadas por betas construidas para recoger la máxima variabilidad posible en los activos del mercado de capitales. Las 8 carteras agrupadas por betas se construyeron a partir de los rendimientos promedio de las carteras que se obtienen ordenando los activos financieros españoles de forma ascendente en dos clases: baja $(\mathrm{L})$ y alta $(\mathrm{H})$ de acuerdo con (y en este orden) el valor de la beta de mercado, la beta asociada al factor de riesgo tamaño (SMB) y la beta asociada al factor de riesgo ratio book-to-market (HML) para una muestra aleatoria de 57 fechas $^{8}$. En segundo lugar otras 2 carteras: la variable "term" que recoge la información del mercado de renta fija a largo/medio y corto plazo, y la variable "def" que recoge un diferencial de insolvencia calculado como el margen entre en rendimiento de la deuda empresarial y la Deuda Pública, aproximándose la primera a través del tipo de interés de las obligaciones de empresa a más de dos años.

Las 5 variables de control, siguiendo a Lamont (2001), establecen relaciones entre los rendimientos de los activos cotizados y los cambios producidos para un conjunto de variables macroeconómicas. Estas variables de control son susceptibles de mejorar la precisión de los parámetros estimados. Dichos parámetros retardados un mes son: los excesos de los rendimientos de la cartera de mercado respecto al activo libre de riesgo (EXM), el factor SMB, el factor HML, el ratio dividendo-precio de la cartera de mercado (div) y el diferencial de tipos (term). La elección de nuestras variables de control viene motivada por

\footnotetext{
${ }^{8}$ La ordenación de los activos se realiza tomando las betas estimadas a partir de una muestra aleatoria de 57 meses anteriores; con esta medida se intentan reducir los problemas de sesgos en la selección (otra alternativa propuesta en Chen, 1983, consiste en separar las observaciones en dos grupos dedicando la primera mitad de la muestra a la construcción de las carteras y la segunda a la estimación de los modelos), sin reducir el número de observaciones de las carteras ni eliminar las estructuras dinámicas en riesgos y primas que se derivan de la estimación.
} 
las evidencias proporcionadas en Font y Grau (2007) sobre la capacidad de la variables EXM, SMB y HML para predecir el crecimiento del PIB, renta y salarios, para el mercado de capitales español.

Paso 3: Obtención de la cartera réplica (R $\triangle \mathrm{PIB}$ : frecuencia mensual). A partir de los coeficientes "b" estimados $(\hat{b})$ en la siguiente regresión (con corrección de errores por Newey y West, 1987) para los datos de una nueva muestra aleatoria de 57 meses anteriores:

$$
\Delta \mathrm{PIB}_{\mathrm{t}, \mathrm{t}+3}=\alpha_{0}+\mathrm{bA}_{\mathrm{t}-1, \mathrm{t}}+\mathrm{mC}_{\mathrm{t}-2, \mathrm{t}-1}+\varepsilon_{\mathrm{t}, \mathrm{t}+3}
$$

donde $\mathrm{A}_{\mathrm{t}-1, \mathrm{t}}$ denota los 10 activos base en $\mathrm{t}, \mathrm{y}_{\mathrm{t}-2, \mathrm{t}-1}$ las 5 variables de control en $\mathrm{t}-1$. Finalmente la serie de la cartera réplica se obtiene de:

$$
\mathrm{R} \Delta \mathrm{PIB}_{\mathrm{t}, \mathrm{t}+1}=\hat{\mathrm{b}} \mathrm{A}_{\mathrm{t}-1, \mathrm{t}}
$$

siendo $\mathrm{R} \Delta \mathrm{PIB}_{\mathrm{t}, \mathrm{t}+1}$ los rendimientos mensuales de la serie $\Delta \mathrm{PIB}_{\mathrm{t}, \mathrm{t}+3}$ para $\mathrm{t}=$ Ene93, Feb-93,...,Dic-11.

El análisis de los resultados estadísticos para la cartera réplica (véase la Tabla 1, Panel C) es de crucial relevancia y merecen especial atención. Observamos que, para el período completo, los ajustes que se detallan para las carteras en posiciones más extremas (HHH, HLH y HLL) aportan información que pone de manifiesto su capacidad predictiva sobre el crecimiento del PIB, siendo esta una de las cuestiones principales, objeto de estudio, del presente trabajo. Efectivamente, estas carteras contribuyen de forma individual y significativa a la predicción del crecimiento del PIB. Particularmente, el estadístico de contraste y su significatividad son más altos para el período de expansión económica. Estos hallazgos mantienen sus características cuando extendemos el estudio al resto de subperiodos considerados. No se observa ningún cambio de signo ni cambios notables en los estadísticos de contraste a excepción del período de crisis financiera-08 donde bajan los niveles de significatividad.

Estos primeros resultados son indicativos, por un lado, de la capacidad predictiva de la cartera réplica construida y su relevancia a la hora de medir los riesgos futuros; y por otro que, la misma es mucho mayor en la fase de bonanza económica respecto a las fases de recesión económica (crisis económica-93 y financiera-08). En el Panel D se observa que los rendimientos para el factor réplica (R $\triangle \mathrm{PIB}$ ) son positivos pero no significativos. La media de los rendimientos se observa más reducida en los períodos de crisis respecto al de expansión económica, siendo el período de crisis financiera-08 el que arroja los valores más reducidos.

\section{METODOLOGÍA APLICADA}

Los modelos de valoración estimados en este artículo y la metodología de 
estimación y contrastación aplicada, son los que se detallan seguidamente.

\subsection{Modelos de valoración propuestos}

En este trabajo se compara la capacidad para explicar los precios de los activos financieros españoles de varios modelos de valoración competitivos: el modelo racional económico (MRE) y el "clásico" modelo de tres factores de Fama y French (1993) extendido con factores de negociación momentum.

Una de nuestras contribuciones diferenciales consiste en incorporar la cartera réplica como fuente de riesgo en un modelo de valoración. Para desarrollar esta metodología nos apoyamos en las evidencias previas vertidas en Vassalou (2003). En consecuencia, diseñamos el primero de ellos, el modelo MRE, que considera el riesgo de mercado y el riesgo asociado al factor réplica del PIB, medido a través de la serie $\mathrm{R} \Delta \mathrm{PIB}$. La ecuación que lo define es:

$$
E\left(r_{j}\right)=\gamma_{0}+\gamma^{m} \beta_{j}^{m}+\gamma^{r \Delta p i b} \beta_{j}^{r \Delta p i b}
$$

donde $E\left(r_{j}\right)$ es el valor esperado de los excesos de rendimientos de un activo $\mathrm{j}$ sobre el activo libre de riesgo del mercado doméstico; $\gamma^{\mathrm{m}}$ es el valor esperado de los excesos de los rendimientos de la cartera de mercado respecto al activo libre de riesgo (prima de mercado) y $\gamma^{\text {rApib }}$ el valor esperado de los rendimientos de la cartera $\mathrm{R} \triangle \mathrm{PIB}$ (prima réplica del PIB); y $\beta_{\mathrm{j}}^{\mathrm{m}}$ es el riesgo beta de activo $\mathrm{j}$ respecto a la cartera de mercado y $\beta_{\mathrm{j}}^{\mathrm{r} \Delta \text { pib }}$ el riesgo beta de activo $\mathrm{j}$ respecto al factor R $\triangle \mathrm{PIB}$. Es interesante notar que el cumplimiento de este modelo y siguientes implica $\gamma_{0}=0$, pero esta ecuación y las dos siguientes al incluir $\gamma_{0}$ permite considerar una formulación del tipo Black (1972) con un rendimiento para el activo cero-beta igual al activo libre de riesgo más $\gamma_{0}$.

La anomalía momentum es persistente a nivel temporal y no puede ser explicada a través del modelo de Fama y French (1993). Para medir el efecto momentum en la valoración de activos, esencialmente nos apoyamos en el trabajo de Carhart (1997) donde propone un modelo que integra cuatro factores de riesgo: mercado, tamaño, ratio book-to-market y momentum. La principal crítica a este modelo es la consideración del factor momentum como factor de riesgo, una alternativa a este modelo explorada en Font y Grau $(2007,2009)$ consiste en añadir a los tres factores de Fama y French (1993) los factores momentum como factores de negociación. Siguiendo esta línea, describimos el modelo de tres factores y momentum $(\mathrm{FF}(\mathrm{m})$ ) que extiende el modelo de Fama y French (1993) (FF) de tres factores de riesgo: mercado, tamaño y ratio book-to-market, aña- 
diendo los efectos momentum (o contrarian) como factores de negociación ${ }^{9}$. El modelo quedaría descrito por la siguiente ecuación:

$$
\mathrm{E}\left(\mathrm{r}_{\mathrm{j}}\right)=\gamma_{0}+\gamma^{\mathrm{m}} \beta_{\mathrm{j}}^{\mathrm{m}}+\gamma^{\mathrm{smb}} \beta_{\mathrm{j}}^{\mathrm{smb}}+\gamma^{\mathrm{hml}} \beta_{\mathrm{j}}^{\mathrm{hml}}+\sum_{\mathrm{k}} \mathrm{g}^{\text {mom_k}} \mathrm{MOM} \_\mathrm{k}
$$

donde $\gamma^{\text {smb }}$ y $\gamma^{\text {hml }}$ son los valores esperados de los rendimientos del factor SMB (prima tamaño) y factor HML, (prima ratio book-to-market) respectivamente; $\beta_{\mathrm{j}}^{\mathrm{smb}}$ y $\beta_{\mathrm{j}}^{\mathrm{hml}}$ son los riesgo beta de activo $j$ respecto a los factores SMB y HML respectivamente; $\mathrm{y} \mathrm{g}^{\text {mom_k }}$ es el coeficiente asociado al efecto (factor de negociación) momentum $k$-ésimo; MOM_k es la cartera efecto momentum $k$ ésima. Este modelo incluye como casos particulares: el modelo CAPM si se acepta la hipótesis $\gamma^{\mathrm{smb}}=0, \gamma^{\mathrm{hml}}=0, \mathrm{~g}^{\text {mom_k}}=0, \forall \mathrm{k}, \mathrm{y}$ el modelo FF si $\mathrm{g}^{\text {mom_k }}$ $=0, \forall \mathrm{k}$.

Han sido muchos los trabajos que han estimado y contrastado varios modelos con factor momentum. Forner y Marhuenda $(2001,2004)$ estudian el efecto contrarian (sobre-reacción), para el mercado que nos ocupa. En el primer trabajo encuentran nula capacidad explicativa para este factor, en cambio para el segundo, la estrategia contrarían a cinco años es, en principio, efectiva, lo cual presta cierto apoyo a la posible existencia de un efecto sobre-reacción en el mercado de capitales español (1963-1997). Si bien este fenómeno fue especialmente fuerte y robusto antes de 1990, se ha debilitado a partir de entonces, desapareciendo prácticamente en la década de los noventa.

Se ha observado a lo largo de la literatura financiera que, en estos últimos años el mercado español parece haberse alineado con el comportamiento de ausencia de momentum detectado, citemos a modo de ejemplo, los trabajos de Forner y Marhuenda, 2006 y Muga y Santamaria, 2007.

Muga y Santamaría (2006) analizan también el efecto momentum y sobre todo, su estabilidad temporal (1991-2004). De acuerdo con los resultados que obtienen concluyen que el efecto momentum aunque significativo, no resulta ser muy persistente ya que parece diluirse con el comienzo de la crisis financiera08 .

\footnotetext{
${ }^{9}$ Se ha optado por un modelo de valoración que incluye los efectos momentum como factores de negociación por dos razones: (i) la ausencia de un consenso en la literatura sobre la naturaleza de riesgo de estos factores (citemos p.e. entre los numerosos trabajos que apoyan una interpretación conductista Jegadeesh y Titman, 1993); y (ii) los resultados de la diagnosis del modelo FF añadiendo el efecto momentum como factor de riesgo, efectivamente la inclusión del momentum como factor de riesgo incrementa sustancialmente el error de estimación (en sesgo y en varianza) respecto al modelo $\mathrm{FF}(\mathrm{m})$ para ambas agrupaciones.
} 


\subsection{Aproximaciones econométricas y metodología}

En la formulación teórica de los modelos, se estiman asumiendo que los primeros y segundos momentos de los rendimientos de los activos son constantes. Esta hipótesis no es realista y por tanto en este trabajo se asume el cumplimiento condicional (no estático) de los modelos propuestos y se implementa para su formulación marginal y posterior estimación el procedimiento escalado ${ }^{10}$ propuesto en Cochrane (1996). Esta metodología condicional es más apropiada atendiendo a la naturaleza cambiante del mercado de capitales, es decir, se va generando continuamente nueva información que es absorbida por los precios cotizados. Este procedimiento escalado permite captar esta naturaleza dinámica a través de una serie de variables de estado (instrumentales) que son indicativas de la evolución del ciclo económico de la economía. En nuestro caso, y ya debidamente justificadas, utilizamos las variables instrumentales div y term.

De acuerdo con esta metodología los modelos MRE y $\mathrm{FF}(\mathrm{m})$ quedarían descritos, en su versión marginal, por las siguientes ecuaciones:

$$
\begin{aligned}
& \mathrm{E}\left(\mathrm{r}_{\mathrm{j}}\right)=\gamma_{0}+\gamma^{\mathrm{m}} \beta_{\mathrm{j}}^{\mathrm{m}}+\gamma^{\mathrm{r} \Delta \mathrm{pib}} \beta_{\mathrm{j}}^{\mathrm{r \Delta pib}}+\gamma^{\mathrm{m} \cdot \mathrm{div}} \beta_{\mathrm{j}}^{\mathrm{m} \cdot \mathrm{div}}+\gamma^{\mathrm{r} \Delta \mathrm{pib} \cdot \mathrm{div}} \beta_{\mathrm{j}}^{\mathrm{r} \Delta \mathrm{pib} \cdot \mathrm{div}}+\gamma^{\mathrm{m} \cdot \mathrm{term}} \beta_{\mathrm{j}}^{\mathrm{m} \cdot \text { term }} \\
& +\gamma^{\text {rapib-term }} \beta_{\mathrm{j}}^{\text {rapib-term }}+\gamma^{\text {div }} \beta_{\mathrm{j}}^{\text {div }}+\gamma^{\text {term }} \beta_{\mathrm{j}}^{\text {term }} \\
& \mathrm{E}\left(\mathrm{r}_{\mathrm{j}}\right)=\gamma_{0}+\gamma^{\mathrm{m}} \beta_{\mathrm{j}}^{\mathrm{m}}+\gamma^{\mathrm{smb}} \beta_{\mathrm{j}}^{\mathrm{smb}}+\gamma^{\mathrm{hml}} \beta_{\mathrm{j}}^{\mathrm{hml}}+\sum_{\mathrm{k}} \mathrm{g}^{\text {mom_k }} \mathrm{MOM} \_\mathrm{k}+\gamma^{\mathrm{m} \cdot \mathrm{div}} \beta_{\mathrm{j}}^{\mathrm{m} \cdot \mathrm{div}} \\
& +\gamma^{\text {smb-div }} \beta_{j}^{\text {smb-div }}+\gamma^{\text {hml-div }} \beta_{j}^{\text {hmld.div }}+\gamma^{\text {m.term }} \beta_{j}^{\text {m.term }}+\gamma^{\text {smb.term }} \beta_{j}^{\text {smb.term }} \\
& +\gamma^{\text {hml-term }} \beta_{j}^{\text {hmlterm }}+\gamma^{\text {div }} \beta_{j}^{\text {div }}+\gamma^{\text {term }} \beta_{j}^{\text {term }}
\end{aligned}
$$

donde $\mathrm{E}\left(\mathrm{r}_{\mathrm{j}}\right)$ es el valor esperado de los excesos de rendimientos de un activo/cartera $j$ sobre el activo libre de riesgo del mercado; $\gamma^{\mathrm{m}}, \gamma^{\mathrm{r} \Delta \mathrm{pib}}, \gamma^{\mathrm{smb}} \mathrm{y}$ $\gamma^{\mathrm{hml}}$ son las primas de riesgo de mercado y asociadas al factor de riesgo asociados a la réplica del PIB, al tamaño y al ratio book-to-market respectivamente; $\beta_{\mathrm{j}}^{\mathrm{m}}, \beta_{\mathrm{j}}^{\mathrm{r} \Delta \mathrm{pib}}, \beta_{\mathrm{j}}^{\mathrm{smb}}$ y $\beta_{\mathrm{j}}^{\mathrm{hml}}$ son los riesgos beta del activo/cartera j respecto a las carteras de mercado y asociadas al factor de riesgo asociado a la réplica del PIB, al tamaño y al ratio book-to-market respectivamente; $\mathrm{g}^{\text {mom_k }}$ es el coeficiente asociado al $k$-ésimo factor de negociación momentum (o contrarian); $\gamma^{\mathrm{F} \cdot \mathrm{I}} \mathrm{y}$ $\beta_{\mathrm{j}}^{\mathrm{F} \cdot \mathrm{I}}, \mathrm{F}=\mathrm{m}, \mathrm{r} \Delta \mathrm{pib}, \mathrm{smb}, \mathrm{hml}, \mathrm{I}=\mathrm{div}$, term tienen la misma interpretación pero

${ }^{10}$ En el proceso escalado de Cochrane (1996) se parte de la expresión en términos de la ecuación de valoración del correspondiente modelo CAPM y se introduce la dinámica sobre los factores de descuento. Otra solución alternativa, propuesta en Dumas y Solnik (1995) y Jagannathan y Wang (1996), consiste en introducir la dinámica directamente sobre las primas de riesgo. 
para los efectos cruzados de los factores de riesgo con las variables instrumentales retardadas un mes; y $\gamma^{\mathrm{I}}$ y $\beta_{\mathrm{j}}^{\mathrm{I}}$, I = div, term son primas y riesgos beta asociados a la variación del ciclo económico recogida por las variables instrumentales retardadas un mes.

Los resultados de este artículo se basan principalmente en la estimación condicional de los modelos MRE (véase ecuación [5]) y FF(m) (véase ecuación [6]) para las agrupaciones de carteras por sector y por tamaño-BM; la cuantificación y comparación de las primas económica relativas a los riesgos asociados a la réplica del PIB, al tamaño y al ratio book-to-market, y del impacto económico asociado a los factores de negociación momentum; y, en definitiva, la comparación entre los modelos de valoración: MRE, FF, FF(m), FF(r $\Delta$ pib) ${ }^{11}$ y CAPM.

En la estimación de los modelos de valoración se aplica la metodología propuesta en Fama y MacBeth (1973). Esta metodología, de uso muy extendido, es idónea para estimar y contrastar los modelos de valoración y analizar la estructura de sección cruzada de los rendimientos de nuestros activos.

Este procedimiento se desarrolla en dos etapas (véanse Ferson y Harvey, 1991, 1999) y se ha implementado en los siguientes términos:

Etapa 1: Obtención de las series condicionales de los riesgos (betas) para cada factor. Se estiman para cada $t(t=1, \ldots, 228$ meses) por $M C O$ los riesgos beta de todos los factores regresando la serie de los excesos de los rendimientos sobre cada uno de los factores considerados ${ }^{12}$, es decir, de forma individual. Como subproducto de la estimación se obtienen en esta primera etapa las series condicionales de los riesgos beta de cada factor.

Etapa 2: Estimación de las primas al riesgo (gammas). Se estiman las primas al riesgo para cada $t$ regresando, conjuntamente, los excesos de los rendimientos de las carteras de cada agrupación sobre las betas correspondientes (estimadas en la primera etapa). Este proceso se lleva a cabo aplicando la metodología SUR (con estimación simultánea de los coeficientes de las primas de riesgo y de la matriz de varianzas-covarianzas del modelo). Como subproducto de la estimación de esta etapa, se obtienen las series condicionales de las primas al riesgo de cada factor.

Dado que nuestro trabajo se sostiene en una metodología condicional de los modelos, y siguiendo la línea de trabajo de implementar técnicas que permitan

${ }^{11}$ El modelo $\mathrm{FF}(\mathrm{r} \Delta$ pib) es el modelo $\mathrm{FF}(\mathrm{m})$ aumentado con el factor $\mathrm{R} \Delta \mathrm{PIB}$.

${ }^{12}$ Otros autores para reducir el efecto de la multicolinealidad en las regresiones de sección cruzada estiman conjuntamente las betas regresando la serie de los excesos sobre el modelo más completo con todos los factores. La ventaja es la reducción de la multicolinealidad pero el inconveniente es que en ese caso se estiman las "betas de cada factor en la parte no explicada por los restantes" y los modelos teóricos no hablan de esas betas. 
incorporar el dinamismo de la información que se va generando en el mercado, seguimos la variante de regresión "rolling beta" de Shanken (1992). Esta técnica permite obtener una serie de riesgos y no un único riesgo representativo de toda la muestra, es decir, una serie para los riesgos (beta) y otra para las primas al riesgo (gamma). Se ha tomado una ventana de observación de 57 datos anteriores que se va desplazando mes a mes, a lo largo de la muestra y va recalculando los citados riesgos para cada $t$. Shanken (1992) solamente lo aplica en la primera etapa, pero en el presente estudio lo extendemos a las dos etapas y por tanto, adquiere la característica de doble procedimiento en "rolling beta".

A partir de los resultados de la segunda etapa se calcula el estimador de las primas para todo el periodo, se realizan los contrastes individuales y conjunto de los parámetros de cada modelo, y se obtienen las series de errores. El error de estimación de cada modelo se calcula a partir de la suma de la media del cuadrado de los errores (errores debidos a la sesgadez del estimador) más la varianza.

Este procedimiento para condicionar los modelos y la flexibilidad del procedimiento para incorporar de forma progresiva los cambios que se van produciendo en el mercado ("rolling beta"), hacen preferible esta metodología frente a la estimación conjunta de ambos grupos de parámetros usando la muestra completa (véase Gibbons, 1982) y el método GMM propuesto en Cochrane (1996). También se ha preferido este procedimiento a la metodología $G A R C H$ multivariante propuesta en De Santis y Gerard (1997) porque, aunque esta última metodología permite calcular las series condicionales de riesgos primas al riesgo (absolutos) de cada factor, asume una estructura dinámica GARCH sobre los excesos de rendimientos que no es adecuada para explicar las series mensuales (véase Tabla 1 donde se aprecia que la estructuras dinámicas de nuestras series son prácticamente inexistentes).

Por otra parte, no podemos pasar por alto el problema de los errores de medida procedentes de los coeficientes betas estimados y fundamentalmente cuando se trate de testear la validez del modelo CAPM. Existe una medida propuesta en Shanken (1992) que consiste en corregir la varianza del estimador. Esta medida de corrección se aplica para argumentar que un riesgo (casi siempre el de mercado) es significativo cuando en los contrastes habituales no lo es debido a errores de medida en la estimación de las betas. En nuestro caso no solamente estimamos los riesgos beta sino, también las primas al riesgo (gammas) y por tanto, si aplicáramos esta corrección las gammas se harían más significativas. En consecuencia, no se realiza porque supondría una sobrecorrección y no precisaríamos argumentos adicionales para que las primas sean significativas. Por otro lado, se han seguido las indicaciones vertidas en Fama y MacBeth (1973) donde proponen agrupar los activos en carteras para poder paliar esta problemática. 
La prima económica asociada a cada factor de riesgo depende de la sensibilidad de cada cartera a las distintas fuentes de riesgo y puede cuantificarse (véase, p.e. De Santis, Gerard y Hillion, 2003) descomponiendo los excesos de los rendimientos totales estimados para cada cartera a partir del correspondiente modelo de valoración.

Concretamente, para estudiar el impacto económico asociado a la réplica del PIB contenido en las acciones (R $\triangle \mathrm{PIB}$ ) se descomponen los excesos de los rendimientos totales estimados para cada cartera a partir del modelo MRE (véase ecuación [3]), en su versión econométrica en las siguientes partes:

Premia mercado: $\quad \gamma^{\mathrm{m}} \beta_{\mathrm{j}}^{\mathrm{m}}+\gamma^{\mathrm{m} \cdot \mathrm{div}} \beta_{\mathrm{j}}^{\mathrm{m} \cdot \mathrm{div}}+\gamma^{\mathrm{m} \cdot \text { term }} \beta_{\mathrm{j}}^{\mathrm{m} \cdot \text { term }}$

Premia réplica del PIB:

$$
\gamma^{\mathrm{r} \Delta \mathrm{pib}} \beta_{\mathrm{j}}^{\mathrm{r} \Delta \text { pib }}+\gamma^{\mathrm{r} \Delta \text { pib-div }} \beta_{\mathrm{j}}^{\mathrm{r} \Delta \text { pib·div }}+\gamma^{\mathrm{r} \Delta \text { pib·term }} \beta_{\mathrm{j}}^{\mathrm{r} \Delta \text { pib.term }}
$$

Premia total:

$$
\gamma_{0}+\gamma^{\mathrm{m}} \beta_{\mathrm{j}}^{\mathrm{m}}+\gamma^{\mathrm{r} \Delta \mathrm{pib}} \beta_{\mathrm{j}}^{\mathrm{r} \Delta \mathrm{pib}}+\gamma^{\mathrm{m} \cdot \mathrm{div}} \beta_{\mathrm{j}}^{\mathrm{m} \cdot \mathrm{div}}+\gamma^{\mathrm{r \Delta pib} \cdot \mathrm{div}} \beta_{\mathrm{j}}^{\mathrm{r} \Delta \mathrm{pib} \cdot \mathrm{div}}
$$

$$
+\gamma^{\mathrm{m} \cdot \text { term }} \beta_{\mathrm{j}}^{\mathrm{m} \cdot \text { term }}+\gamma^{\mathrm{r} \Delta \mathrm{pib} \cdot \mathrm{term}} \beta_{\mathrm{j}}^{\mathrm{r} \Delta \mathrm{pib} \cdot \mathrm{term}}+\gamma^{\mathrm{div}} \beta_{\mathrm{j}}^{\text {div }}+\gamma^{\text {term }} \beta_{\mathrm{j}}^{\text {term }}
$$

Para el modelo $\mathrm{FF}(\mathrm{m})$ (véase ecuación [4]) en las siguientes partes:

Premia mercado: $\quad \gamma^{\mathrm{m}} \beta_{\mathrm{j}}^{\mathrm{m}}+\gamma^{\mathrm{m} \cdot \mathrm{div}} \beta_{\mathrm{j}}^{\mathrm{m} \cdot \mathrm{div}}+\gamma^{\mathrm{m} \cdot \text { term }} \beta_{\mathrm{j}}^{\mathrm{m} \cdot \text { term }}$

Premia tamaño: $\quad \gamma^{\text {smb }} \beta_{j}^{\text {smb }}+\gamma^{\text {smb.div }} \beta_{j}^{\text {smb-div }}+\gamma^{\text {smb.term }} \beta_{j}^{\text {smb.term }}$

Premia ratio book-

to-market:

$$
\gamma^{\mathrm{hml}} \beta_{\mathrm{j}}^{\mathrm{hml}}+\gamma^{\mathrm{hml} \cdot \mathrm{div}} \beta_{\mathrm{j}}^{\mathrm{hml} \cdot \mathrm{div}}+\gamma^{\mathrm{hml} \cdot \text { term }} \beta_{\mathrm{j}}^{\mathrm{hml} \cdot \text { term }}
$$

Impacto económico

efecto momentum:

$$
\sum_{\mathrm{k}} \mathrm{g}^{\text {mom_k}} \mathrm{MOM} \_\mathrm{k}
$$$$
\gamma_{0}+\gamma^{\mathrm{m}} \beta_{\mathrm{j}}^{\mathrm{m}}+\gamma^{\mathrm{smb}} \beta_{\mathrm{j}}^{\mathrm{smb}}+\gamma^{\mathrm{hml}} \beta_{\mathrm{j}}^{\mathrm{hml}}+\sum_{\mathrm{k}} \mathrm{g}^{\text {mom_k}} \mathrm{MOM}_{-} \mathrm{k}
$$

Premia total:

$$
\begin{aligned}
& +\gamma^{\text {m.div }} \beta_{j}^{\text {m.div }}+\gamma^{\text {smb-div }} \beta_{j}^{\text {smb-div }}+\gamma^{\text {hml-div }} \beta_{j}^{\text {hml.div }}+\gamma^{\text {m.term }} \beta_{j}^{\text {m.term }} \\
& +\gamma^{\text {smb-term }} \beta_{j}^{\text {smb.term }}+\gamma^{\text {hml.term }} \beta_{j}^{\text {hmlterm }}+\gamma^{\text {div }} \beta_{j}^{\text {div }}+\gamma^{\text {term }} \beta_{j}^{\text {term }}
\end{aligned}
$$

cada uno de los componentes que los integran, y extensible a todos los modelos, se evalúan usando las series de riesgos y primas de riesgo condicionales obtenidas en la primera y segunda fase de la estimación por Fama y MacBeth (1973).

Para las primas económicas calculamos su valor medio y su error estándar. Este cálculo se efectúa para la muestra completa y tres subperíodos y consiste en regresar las series obtenidas para las primas sobre un término constante y 
seguidamente aplicamos la corrección por heterocedasticidad y autocorrelación de Newey y West (1987).

Adicionalmente, se ha estimado y contrastado el modelo $\mathrm{FF}(\mathrm{m})$ aumentado que se obtienen añadiendo el factor réplica del PIB (denotado por $\mathrm{FF}(\mathrm{r} \Delta \mathrm{pib})$ ) para analizar respectivamente la validez de las explicaciones racionales de los factores tamaño, ratio book-to-market y momentum propuestas. Su estructura es la siguiente ${ }^{13}$ :

$$
\begin{aligned}
& \mathrm{E}\left(\mathrm{r}_{\mathrm{j}}\right)=\gamma_{0}+\gamma^{\mathrm{m}} \beta_{\mathrm{j}}^{\mathrm{m}}++\gamma^{\mathrm{r} \Delta \mathrm{pib}} \beta_{\mathrm{j}}^{\mathrm{r} \Delta \mathrm{pib}} \gamma^{\mathrm{smb}} \beta_{\mathrm{j}}^{\mathrm{smb}}+\gamma^{\mathrm{hml}} \beta_{\mathrm{j}}^{\mathrm{hml}}+\sum_{\mathrm{k}} \mathrm{g}^{\mathrm{mom} \_\mathrm{k}} \mathrm{MOM} \_\mathrm{k}
\end{aligned}
$$



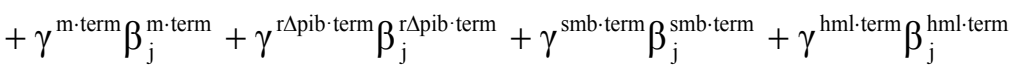

$$
\begin{aligned}
& +\gamma^{\text {div }} \beta_{\mathrm{j}}^{\text {div }}+\gamma^{\text {term }} \beta_{\mathrm{j}}^{\text {term }}
\end{aligned}
$$

\section{RESULTADOS EMPÍRICOS}

Se dedica este apartado al análisis de los resultados del artículo. Primeramente, se estudia la capacidad del factor réplica del PIB para explicar los rendimientos de los activos españoles en sección cruzada, mediante la estimación del modelo MRE (véase ecuación [5]) y cuantificando el impacto económico del mismo a partir de su prima económica. En segundo lugar, se comprueba, estimando el modelo $\mathrm{FF}(\mathrm{m})$ (véase ecuación [6]), que el mercado paga primas significativas por los riesgos que derivan de los factores tamaño y ratio book-tomarket y la contribución conjuntamente significativa de los cuatro efectos momentum considerados para explicar los rendimientos de los activos españoles; valorando, a continuación, el impacto económico de estos factores de riesgo y efectos momentum. En tercer lugar, se comparan los modelos de valoración presentados junto con sus modelos anidados. Y finaliza el estudio analizando la naturaleza racional de los efectos tamaño, ratio book-to-market y momentum estableciendo la relación de los mismos con el factor de riesgo réplica del PIB.

\subsection{El factor réplica del PIB en la valoración de activos financieros}

Comenzamos analizando los resultados estadísticos de la estimación y contraste del modelo MRE (véase ecuación [5]) y posteriormente analizaremos las implicaciones en materia de valoración. Para todos los casos se acepta la hipótesis de insesgadez ${ }^{14}$ de los errores (obsérvese en la Figura 1 que los errores

\footnotetext{
${ }^{13}$ Para evitar redundancias en la notación del artículo, se ha omitido la explicación de las variables que integran este modelo dado que en los precedentes ya se ha indicado esta información.

${ }^{14} \mathrm{El}$ contraste de insesgadez es: $\mathrm{H}_{0}$ : $\mathrm{E}(\varepsilon)=0$, se ha calculado a partir de la suma de la media del cuadrado de los errores (errores debidos a la sesgadez del estimador) más la varianza.
} 
estimados están dentro de la banda de confianza). Los resultados, para el período completo, presentados en la Tabla 2 (Panel B) permiten rechazar la hipótesis conjunta de riesgos instrumentales nulos, en conformidad con la aproximación condicional asumida en este trabajo y por tanto, queda más que justificada la idoneidad de la metodología de estimación condicional elegida. El rechazo de la hipótesis conjunta de que todos los riesgos sean iguales entre sí e iguales a cero, manifiestan la capacidad explicativa del modelo MRE. Los resultados también destacan la significatividad conjunta de las primas de mercado para las dos agrupaciones de carteras, y el error de estimación supera al error del modelo CAPM (véase la Tabla 2: Panel A.1 y B.1).

Figura 1

Representación de los errores de estimación de varios modelos

Panel A: Carteras por Sector
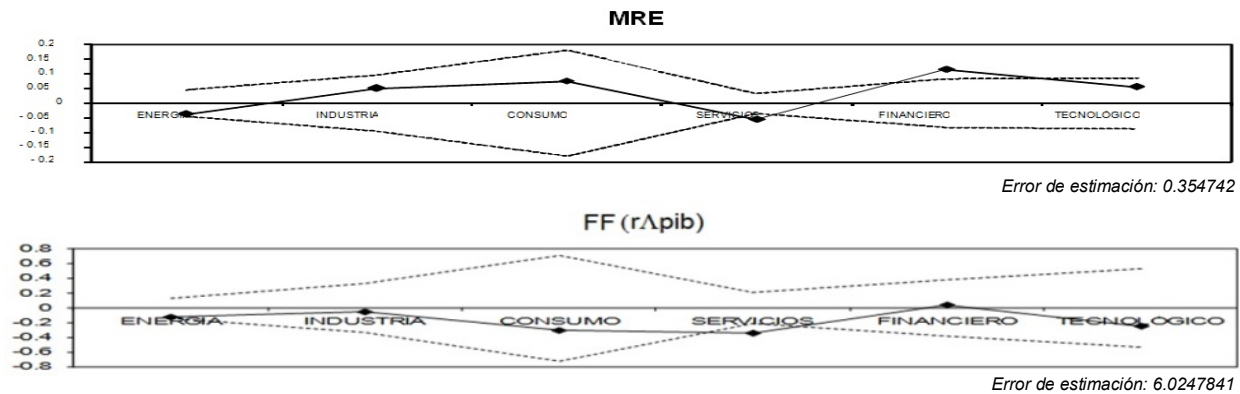

$F F(m)$
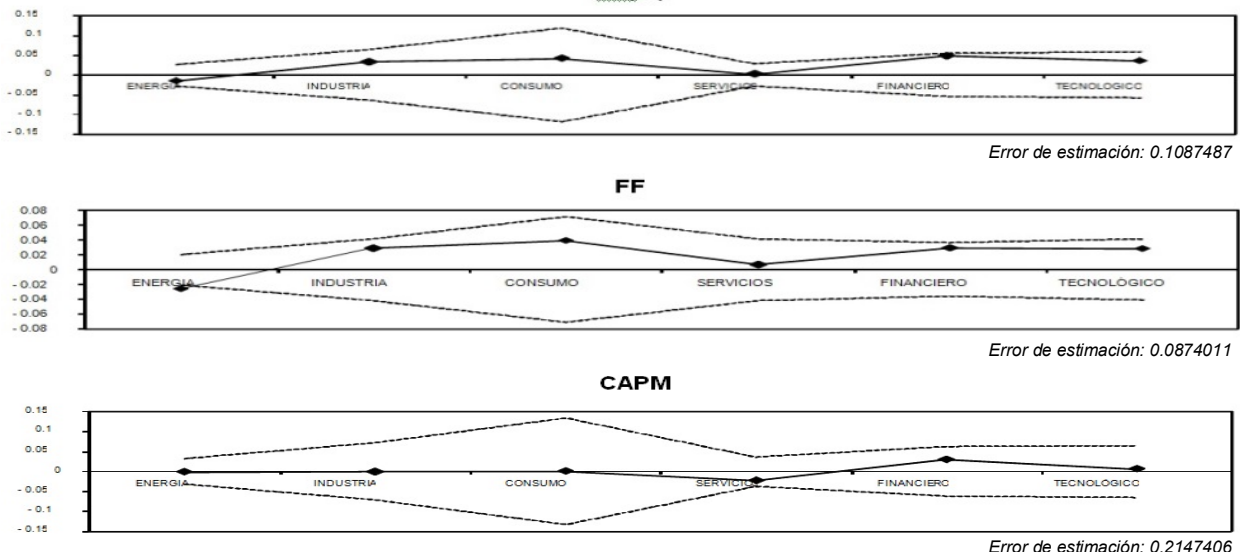
Figura 1 (continuación)

Representación de los errores de estimación de varios modelos

Panel B: Carteras por Tamaño-BM

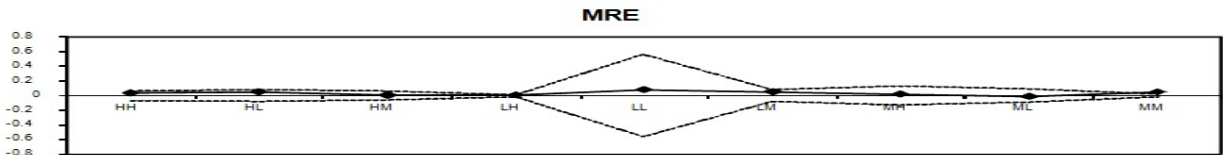

Error de estimación: 1.409978
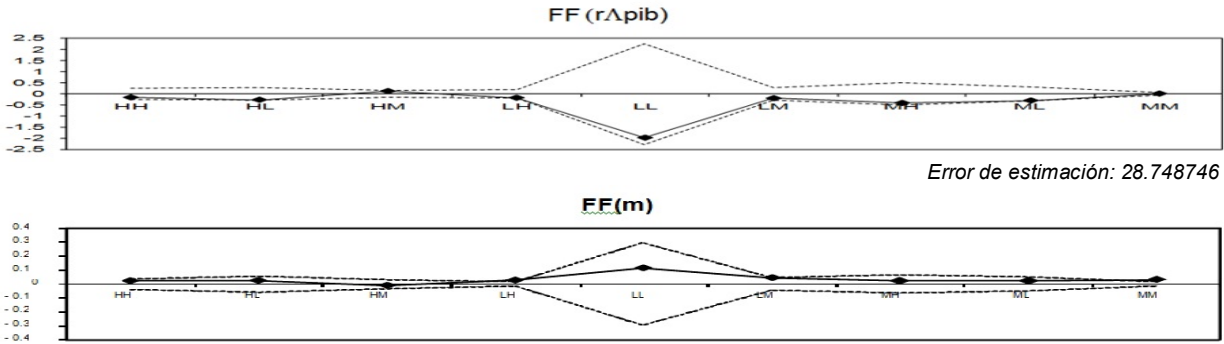

Error de estimación: 0.381146

FF

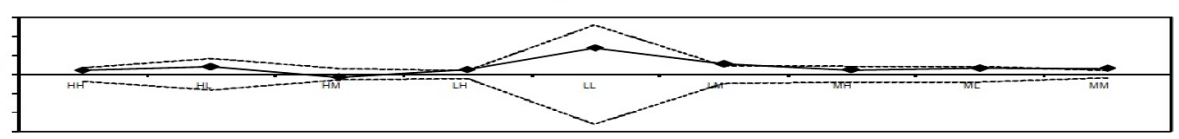

Error de estimación: 0.3014784

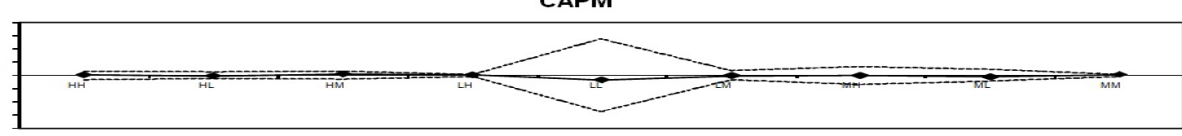

Error de estimación: 1.4784108

La Figura representa los errores de estimación de los modelos MRE, FF(r $\Delta p i b), F F(m)$, FF y CAPM junto con la banda de confianza \pm 2 desviaciones típicas del error de estimación para las dos agrupaciones de carteras.

Fuente: Elaboración propia.

En relación al factor de riesgo asociado a la réplica del PIB, $\mathrm{R} \Delta \mathrm{PIB}$, las primas asociadas a estos riesgos son significativas de forma conjunta y de forma individual para las dos agrupaciones. La cuantía de las primas $-0.5947 \%$ y $-0.1785 \%$ mensual para las agrupaciones por sector y tamaño-BM, son también indicadores de la importancia del factor en la valoración de activos financieros. Estos resultados son consistentes con los presentados por Vassalou (2003) que también obtiene primas significativas (al 5\%) para este factor con datos trimestrales estimando los rendimientos de las 25 carteras de Fama-French (1993) en el periodo comprendido entre enero 1953 y diciembre 1998.

Determinada la significatividad de las primas asociadas al factor réplica del PIB el siguiente paso es cuantificar la magnitud económica de las mismas y, en consecuencia, medir el error cometido cuando se aplica en la valoración el modelo CAPM. Los resultados de la Tabla 3: Panel A, subrayan la relevancia económica de la prima de riesgo asociada a la réplica del PIB para las dos agru- 
paciones y periodo completo y subperíodos considerados. Atendiendo a estos resultados, asumir una valoración basada en el modelo CAPM supondría una incorrecta valoración de nuestros activos financieros para la mayoría de las carteras consideradas en el período completo y subperíodos.

Tabla 2

Estimación y contrastación de los modelos MRE y $F F(m)$

\begin{tabular}{|c|c|c|c|c|c|c|c|c|c|c|}
\hline \multicolumn{11}{|c|}{ Panel A.1: Modelo MRE } \\
\hline & $\gamma_{0}$ & $\gamma^{\mathrm{m}}$ & $\gamma^{\text {rapib }}$ & $\gamma^{\text {m-div }}$ & $\gamma^{\text {rapib -div }}$ & $\gamma^{\mathrm{m} \cdot \mathrm{tem}}$ & $\gamma^{\text {rspib term }}$ & $\gamma^{\text {div }}$ & $\gamma^{\text {tem }}$ & $\begin{array}{c}\text { Error } \\
\text { estimación }\end{array}$ \\
\hline Carteras por Sector & $0.016784^{\star \star}$ & 0.001474 & $-0.005947^{\star \star}$ & -0.001854 & $0.0040784^{\star \star}$ & $0.00000147^{*}$ & $-0.00000241^{\star \star}$ & $-0.002174^{\star *}$ & -0.00000421 & 0.354742 \\
\hline Carteras tamaño-BM & $0.014781^{* \star}$ & -0.005984 & $-0.001785^{\star \star}$ & -0.001597 & $0.0036712^{\star \star}$ & $-0.00000312^{* *}$ & $-0.000000497^{\wedge}$ & $0.001291^{\star \star}$ & 0.00000457 & 1.409978 \\
\hline \multicolumn{11}{|c|}{ Panel A.2: Modelo FF-m } \\
\hline & $\gamma_{0}$ & $\gamma^{m}$ & $\gamma^{\mathrm{smb}}$ & $\gamma^{\mathrm{hml}}$ & $\mathbf{g}^{\text {mom3 }}$ & $\mathrm{g}^{\text {mom6 }}$ & $\mathbf{g}^{\text {mom9 }}$ & $\mathbf{g}^{\text {mom12 }}$ & $\gamma^{\text {m.div }}$ & $\gamma^{\text {smb-div }}$ \\
\hline Carteras por Sector & $0.037487^{\star \star}$ & -0.003487 & 0.034791 & $-0.021247^{\star *}$ & $-0.195474^{* *}$ & $-0.098744^{* *}$ & $0.1400124^{\star \star}$ & $-0.2874112^{* *}$ & -0.0030009 & $-0.018742^{* *}$ \\
\hline \multirow[t]{2}{*}{ Carteras tamaño-BM } & $0.009784^{\star \star}$ & $-0.009871^{\wedge}$ & -0.005178 & 0.003587 & $-0.097447^{\star \star}$ & $-0.068741^{* *}$ & $0.0974511^{\star \star}$ & $-0.124787^{\star \star}$ & -0.0012748 & $-0.011478^{* *}$ \\
\hline & $\gamma^{\text {hml-div }}$ & $\gamma^{\mathrm{m} \cdot \mathrm{tem}}$ & $\gamma^{\text {smb-term }}$ & $\gamma^{\mathrm{hml} \cdot \mathrm{tem}}$ & $\gamma^{\text {div }}$ & $\gamma^{\text {tem }}$ & $\begin{array}{c}\text { Error } \\
\text { estimación }\end{array}$ & & & \\
\hline Carteras por Sector & $0.006783^{\star *}$ & $-0.0000067^{\star *}$ & 0.00000148 & $-0.0000091^{\star *}$ & $-0.001805^{* *}$ & -0.00000712 & 0.187487 & & & \\
\hline Carteras tamaño-BM & $0.002057^{\star}$ & $-0.0000048^{* *}$ & $0.00000708^{* *}$ & $0.00000172^{*}$ & -0.000621 & $-0.0000375^{\star \star}$ & 0.381146 & & & \\
\hline
\end{tabular}

Panel B: Contrastes de especificación para los modelos MRE y FF(m)

Panel B.1: Modelo MRE

\begin{tabular}{|c|c|c|}
\hline $\mathrm{H}_{0}$ & Sector & Tamaño-BM \\
\hline 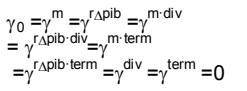 & $3087.241^{\star *}$ & $2074.831^{\star *}$ \\
\hline$\gamma^{m}=\gamma^{m \cdot d i v}=\gamma^{m \cdot t e r m}=0$ & $49.7417^{\star \star}$ & $92.7457^{\star \star}$ \\
\hline $\begin{array}{l}\gamma^{r \Delta \text { pib }}=\gamma^{r_{\Delta} \text { pib div }}=\gamma^{{ }^{r} \Delta \text { pib term }}=0 \\
\gamma^{\text {div }}=\gamma^{\text {term }}=0\end{array}$ & $\begin{array}{l}1012.747^{\star \star} \\
64.7547^{\star \star}\end{array}$ & $\begin{array}{l}209.1487^{\star *} \\
21.4571^{\star *}\end{array}$ \\
\hline
\end{tabular}

\section{Panel B.2: Modelo FF(m)}

\begin{tabular}{|c|c|c|}
\hline $\mathrm{H}_{0}$ & Sector & $\begin{array}{c}\text { Tamaño- } \\
\text { BM }\end{array}$ \\
\hline  & $18742.5^{\star \star}$ & $7315.74^{\star \star}$ \\
\hline$=\gamma^{m \cdot t e r m}=0$ & $56.78411^{* *}$ & $77.7419^{* *}$ \\
\hline$\gamma^{\text {smb }}=\gamma^{\text {smb div }}=\gamma^{\text {smb term }}=0$ & $350.7457^{* *}$ & $491.784^{\star *}$ \\
\hline$\gamma^{\mathrm{hml}}=\gamma^{\mathrm{hml} \cdot \mathrm{div}}=\gamma^{\mathrm{hml} \cdot \operatorname{term}}=0$ & $801.124^{* *}$ & $109.784^{\star *}$ \\
\hline $\mathrm{g}^{\mathrm{mom} 3}=\mathrm{g}^{\mathrm{mom} 6}=\mathrm{g}^{\mathrm{mom} 9}=\mathrm{g}^{\mathrm{mom} 12}=0$ & $135.701^{* *}$ & $287.745^{\star *}$ \\
\hline$\gamma^{\text {div }}=\gamma^{\text {term }}=0$ & $30.7411^{* *}$ & $16.7454^{* *}$ \\
\hline
\end{tabular}

La Tabla presenta, para el periodo completo en el panel A.1 los resultados de la estimación del modelo MRE para las carteras sectoriales y tamaño-BM: primas al riesgo estimadas, su nivel de significatividad y el error de estimación del modelo; $y$ en el Panel B los contrastes de Wald de una selección de hipótesis de interés junto con sunivel de significatividad. Los paneles A.2 y B.2, reproducen la misma información referida a la estimación del modelo $F F(m)$.

Nivel de significatividad de los contrastes: $10 \%\left({ }^{\wedge}\right), 5 \%\left({ }^{*}\right)$ y $1 \%\left(^{* *}\right)$.

Fuente: Elaboración propia.

Analicemos las implicaciones y el alcance de estas evidencias. Atendiendo al número de carteras significativas y su nivel de significatividad, se aprecia que el peligro de infra/sobrevaloración es mucho mayor en las carteras agrupadas por tamaño-BM, por tanto, la agrupación sectorial muestra un nivel de sensibilidad más reducido. Efectivamente, no incluir el factor réplica del PIB en la valoración de los activos implicaría una incorrecta valoración de las carteras, extensible a todos los subperiodos y muestra completa, siendo el más potencialmente castigado el perteneciente a la crisis financiera-08. La etapa de crisis económica-93, ofrece niveles de significatividad más bajos, por tanto el efecto que ha 
tenido, en materia de valoración, no ha impactado tan alarmantemente en los rendimientos de los activos financieros. En la etapa de expansión económica se ha mostrado más moderada.

\section{Tabla 3}

Primas económicas de los factores para los modelos MRE y FF(m)

\section{Panel A: Primas económicas modelo MRE}

Panel A.1: Carteras por Sector

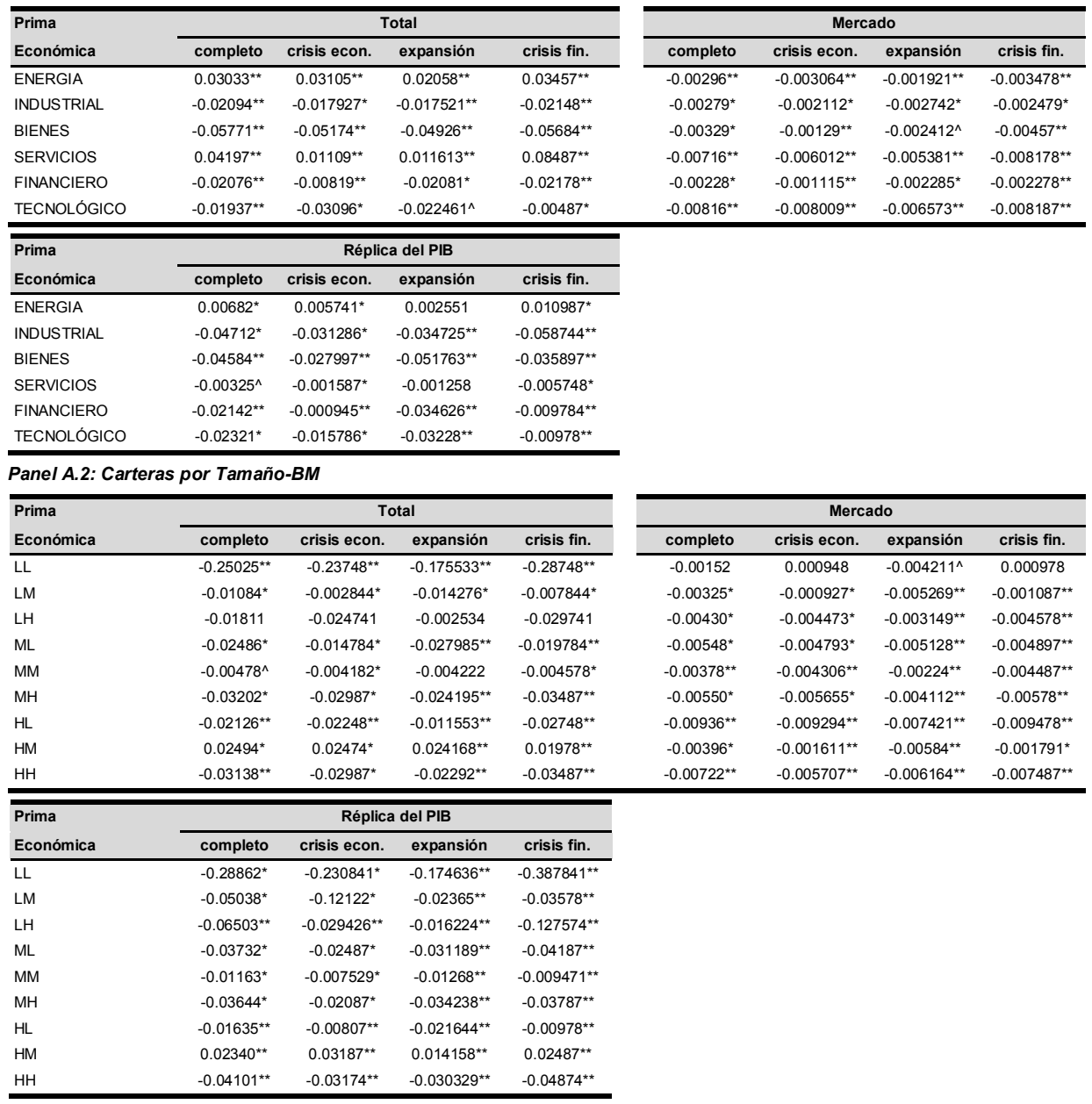


Tabla 3 (continuación)

Primas económicas de los factores para los modelos MRE y $\mathrm{FF}(\mathrm{m})$

Panel B: Primas económicas e impacto económico del factor momentun en el modelo FF(m)

\section{Panel B.1: Carteras por Sector}

\begin{tabular}{|c|c|c|c|c|}
\hline \multirow{2}{*}{$\begin{array}{l}\text { Prima } \\
\text { Económica }\end{array}$} & \multicolumn{4}{|c|}{ Total } \\
\hline & completo & crisis econ. & expansión & crisis fin. \\
\hline ENERGIA & $0.08395^{\star \star}$ & $0.01548^{*}$ & $0.02057^{\star \star}$ & $0.00974^{\star *}$ \\
\hline INDUSTRIAL & $-0.05339^{\star \star}$ & $-0.007873^{*}$ & $-0.01209^{*}$ & $-0.00874^{*}$ \\
\hline BIENES & $-0.08881^{\text {**}}$ & $-0.003233^{\star \star}$ & $-0.03136^{\star \star}$ & $-0.0041^{\star \star}$ \\
\hline SERVICIOS & $0.10564^{*}$ & $0.023895^{\star \star}$ & $0.0154^{\star *}$ & $0.02471^{*}$ \\
\hline FINANCIERO & $-0.05164^{*}$ & $-0.008915^{\star}$ & $-0.01028^{\wedge}$ & $-0.00973^{*}$ \\
\hline TECNOLÓGICO & $-0.05342^{\wedge}$ & $-0.007925^{\wedge}$ & $-0.01208^{\wedge}$ & $-0.00874^{\wedge}$ \\
\hline \multicolumn{5}{|l|}{ Prima } \\
\hline & completo & crisis econ. & expansión & crisis fin. \\
\hline ENERGIA & 0.02135 & 0.003353 & $0.00409^{\wedge}$ & $0.00444^{\wedge}$ \\
\hline INDUSTRIAL & $-0.15784^{*}$ & $-0.03202^{\star}$ & $-0.02645^{\star \star}$ & $-0.03411^{*}$ \\
\hline BIENES & $-0.18748^{\star *}$ & $-0.0389^{* *}$ & $-0.03073^{* *}$ & $-0.0410^{* *}$ \\
\hline SERVICIOS & $-0.01916^{\wedge}$ & -0.008923 & 0.003611 & $-0.01101^{*}$ \\
\hline FINANCIERO & $-0.19446^{*}$ & $-0.04031^{*}$ & $-0.03121^{\star \star}$ & $-0.0435^{\star \star}$ \\
\hline TECNOLÓGICO & $-0.11361^{*}$ & $-0.00551^{*}$ & $-0.03715^{\star *}$ & $-0.0087^{\star \star}$ \\
\hline \multirow{2}{*}{$\begin{array}{l}\text { Prima } \\
\text { Económica } \\
\end{array}$} & \multicolumn{4}{|c|}{ Total + Ratio book-to-market } \\
\hline & completo & crisis econ. & expansión & crisis fin. \\
\hline ENERGIA & 0.03943 & 0.00379 & $0.01093^{\wedge}$ & $0.00475^{\wedge}$ \\
\hline INDUSTRIAL & $-0.18980^{* *}$ & $-0.0291^{* *}$ & $-0.0374^{\star \star}$ & $-0.0384^{\star *}$ \\
\hline BIENES & $-0.34230^{\star *}$ & $-0.0721^{* *}$ & $-0.0511^{\star \star}$ & $-0.0814^{\star *}$ \\
\hline SERVICIOS & -0.00483 & $-0.00028^{\wedge}$ & -0.00117 & -0.00093 \\
\hline FINANCIERO & $-0.16668^{\star \star}$ & $-0.0166^{\star *}$ & $-0.0419^{\star \star}$ & $-0.0259^{\star \star}$ \\
\hline TECNOLÓGICO & $-0.19118^{*}$ & $-0.0296^{\star}$ & $-0.0374^{\star \star}$ & $-0.0389^{*}$ \\
\hline \multicolumn{2}{|c|}{ Impacto Económico Momentum } & -0.002309 & & \\
\hline
\end{tabular}

\section{Panel B.2: Carteras por Tamaño-BM}

\begin{tabular}{|c|c|c|c|c|}
\hline \multirow{2}{*}{$\begin{array}{l}\text { Prima } \\
\text { Económica }\end{array}$} & \multicolumn{4}{|c|}{ Total } \\
\hline & completo & crisis econ. & expansión & crisis fin \\
\hline$\overline{\mathrm{LL}}$ & $-0.61180^{* \star}$ & $-0.0831^{\star *}$ & $-0.1454^{\star \star}$ & $-0.0944^{* *}$ \\
\hline LM & $-0.05540^{* *}$ & $-0.0042^{\star *}$ & $-0.0097^{* *}$ & $-0.0154^{* *}$ \\
\hline $\mathrm{LH}$ & -0.04394 & -0.00012 & $-0.01160^{\wedge}$ & -0.00847 \\
\hline $\mathrm{ML}$ & $-0.14725^{* *}$ & $-0.0199^{\star *}$ & $-0.0291^{\star *}$ & $-0.0312^{\star \star}$ \\
\hline MM & -0.02437 & -0.00223 & -0.00527 & -0.00512 \\
\hline $\mathrm{MH}$ & $-0.03268^{*}$ & $-0.00125^{\star}$ & $-0.0083^{* \star}$ & $-0.0061^{* \star}$ \\
\hline $\mathrm{HL}$ & -0.02825 & -0.00715 & -0.0083 & -0.0002 \\
\hline $\mathrm{HM}$ & $0.16783^{* *}$ & $0.03945^{\star *}$ & $0.02888^{* *}$ & $0.0321^{* *}$ \\
\hline $\mathrm{HH}$ & $-0.10289^{*}$ & $-0.01422^{\star}$ & $-0.02037^{\star}$ & -0.02157 \\
\hline Prima & \multicolumn{4}{|c|}{ Tamaño } \\
\hline Económica & completo & crisis econ. & expansión & crisis fin \\
\hline $\mathrm{LL}$ & $-0.82525^{\star *}$ & $-0.1433^{\star *}$ & $-0.1479^{\star \star}$ & $-0.1784^{\star \star}$ \\
\hline LM & $-0.14038^{* *}$ & $-0.0207^{\star \star}$ & $-0.0268^{* \star}$ & $-0.0301^{* *}$ \\
\hline $\mathrm{LH}$ & $-0.13598^{* *}$ & $-0.0251^{\star \star}$ & $-0.0202^{\star \star}$ & $-0.0345^{\star \star}$ \\
\hline $\mathrm{ML}$ & $-0.12683^{* *}$ & $-0.0105^{\star *}$ & $-0.0326^{* *}$ & $-0.0199^{\star *}$ \\
\hline MM & $-0.11740^{* *}$ & $-0.0218^{* *}$ & $-0.0164^{\star \star}$ & $-0.0312^{* \star}$ \\
\hline $\mathrm{MH}$ & $-0.15473^{* *}$ & $-0.0052^{\star \star}$ & $-0.0316^{\star \star}$ & $-0.0403^{\star \star}$ \\
\hline $\mathrm{HL}$ & -0.01012 & -0.00165 & -0.002764 & -0.000891 \\
\hline $\mathrm{HM}$ & $0.09081^{\star \star}$ & $0.04384^{* *}$ & $0.01267^{\star \star}$ & $0.00874^{* \star}$ \\
\hline $\mathrm{HH}$ & $-0.13708^{\star *}$ & $-0.0096^{\star \star}$ & $-0.0267^{\star \star}$ & $-0.0347^{\star \star}$ \\
\hline
\end{tabular}

\begin{tabular}{|c|c|c|c|}
\hline \multicolumn{4}{|c|}{ Mercado } \\
\hline completo & crisis econ. & expansión & crisis fin. \\
\hline$-0.0635^{\star \star}$ & $-0.0102^{* *}$ & $-0.01031^{* *}$ & $-0.0157^{\star \star}$ \\
\hline$-0.01781^{*}$ & $0.00083^{*}$ & $-0.004186^{*}$ & $-0.00467^{*}$ \\
\hline$-0.00385^{\wedge}$ & $0.00453^{*}$ & $-0.002671^{\wedge}$ & $-0.00097^{\wedge}$ \\
\hline$-0.02548^{*}$ & $-0.0002^{*}$ & $-0.00612^{* *}$ & $-0.0057^{\star \star}$ \\
\hline$-0.01871^{\star}$ & $-0.00737^{*}$ & $-0.003225^{*}$ & $-0.00187^{\star}$ \\
\hline$-0.03932^{\star}$ & $-0.00352^{\star}$ & $-0.008007^{*}$ & $-0.00902^{*}$ \\
\hline \multicolumn{4}{|c|}{ Ratio book-to-market } \\
\hline completo & crisis econ. & expansión & crisis fin. \\
\hline 0.00670 & $5.9 \mathrm{E}-05$ & $0.00211^{\wedge}$ & 0.00095 \\
\hline-0.04387 & $-0.00549^{\star *}$ & $-0.0108^{* *}$ & $-0.0065^{\star *}$ \\
\hline-0.06876 & $-0.00008^{* *}$ & $-0.0274^{* *}$ & $-0.0001^{* *}$ \\
\hline-0.01443 & $-0.000309^{\wedge}$ & $-0.00502^{\wedge}$ & $-0.0009^{* *}$ \\
\hline-0.04309 & $-0.004391^{*}$ & $-0.0117^{\star *}$ & $-0.0054^{* *}$ \\
\hline-0.06917 & $-0.014691^{*}$ & $-0.0108^{* \star}$ & $-0.0157^{\star *}$ \\
\hline
\end{tabular}

\begin{tabular}{|c|c|c|c|}
\hline \multicolumn{4}{|c|}{ Mercado } \\
\hline completo & crisis econ. & expansión & crisis fin. \\
\hline-0.01449 & -0.0044 & -0.000199 & -0.00548 \\
\hline$-0.0293^{* *}$ & $-0.0044^{* *}$ & $-0.00612^{\star \star}$ & $-0.0055^{* *}$ \\
\hline$-0.0190^{\star \star}$ & $-0.0025^{* *}$ & $-0.00408^{\star *}$ & $-0.0036^{* *}$ \\
\hline$-0.03361^{*}$ & $-0.00597^{\star}$ & $-0.00612^{*}$ & $-0.00705^{\star}$ \\
\hline$-0.01774^{\star}$ & $0.0004^{*}$ & $-0.00678^{\star \star}$ & $-0.00068^{*}$ \\
\hline$-0.03634^{*}$ & $-0.00979^{\star}$ & $-0.003012^{*}$ & $-0.01087^{*}$ \\
\hline$-0.0280^{* *}$ & $-0.0041^{* *}$ & $-0.00451^{* *}$ & $-0.0072^{* *}$ \\
\hline$-0.0329^{* *}$ & $-0.0054^{\star *}$ & $-0.00645^{\star \star}$ & $-0.0065^{* *}$ \\
\hline$-0.0443^{* *}$ & $-0.0090^{* *}$ & $-0.00703^{\star *}$ & $-0.0101^{* *}$ \\
\hline
\end{tabular}


Tabla 3 (continuación)

Primas económicas de los factores para los modelos MRE y $\mathrm{FF}(\mathrm{m})$

Panel B.2: Carteras por Tamaño-BM (continuación)

\begin{tabular}{|c|c|c|c|c|c|c|c|c|}
\hline \multirow{2}{*}{$\begin{array}{l}\text { Prima } \\
\text { Económica }\end{array}$} & \multicolumn{4}{|c|}{ Ratio book-to-market } & \multicolumn{4}{|c|}{ Tamaño + Ratio book-to-market } \\
\hline & completo & crisis econ. & expansión & crisis fin. & completo & crisis econ. & expansión & crisis fin. \\
\hline$\overline{L L}$ & $-0.22405^{*}$ & $-0.0099^{*}$ & $-0.0201^{\star \star}$ & $-0.0994^{*}$ & $-0.6043^{\star \star}$ & $-0.1157^{\star \star}$ & $-0.15789^{\star \star}$ & $-0.09874^{* \star}$ \\
\hline LM & $-0.02543^{*}$ & $-0.0052^{\star}$ & $-0.0046^{* *}$ & $-0.0063^{*}$ & $-0.1620^{* *}$ & $-0.0298^{* *}$ & $-0.03384^{\star \star}$ & $-0.03687^{* *}$ \\
\hline LH & -0.00158 & -0.00011 & -0.00060 & -0.00015 & $-0.0927^{\star \star}$ & $-0.0157^{\star \star}$ & $-0.02121^{\star \star}$ & $-0.01974^{\star *}$ \\
\hline ML & $-0.01818^{\star *}$ & $-0.0012^{\star *}$ & $-0.0077^{\star \star}$ & $-0.0009^{\star *}$ & $-0.1430^{\star *}$ & $-0.0445^{* \star}$ & $-0.04074^{\star \star}$ & $-0.00978^{\star \star}$ \\
\hline MM & $-0.00580^{*}$ & $-0.0006^{\star}$ & $-0.0019^{*}$ & $-0.0008^{\star}$ & $-0.1112^{\star \star}$ & $-0.0195^{* \star}$ & $-0.02147^{\star \star}$ & $-0.02784^{\star \star}$ \\
\hline MH & $-0.01855^{\star \star}$ & $-0.0031^{* *}$ & $-0.0037^{\star \star}$ & $-0.0046^{* *}$ & $-0.1144^{\star \star}$ & $-0.0156^{\star \star}$ & $-0.03815^{\star \star}$ & $-0.01287^{\star *}$ \\
\hline $\mathrm{HL}$ & $-0.12910^{* *}$ & $-0.0314^{* \star}$ & $-0.0128^{* *}$ & $-0.0412^{* *}$ & $-0.05819^{*}$ & $-0.0099^{* \star}$ & $-0.01645^{*}$ & $-0.00879^{*}$ \\
\hline HM & 0.00716 & 0.00337 & 0.00094 & $0.00109^{*}$ & $0.0885^{\star *}$ & $0.0144^{* *}$ & $0.01302^{* \star}$ & $0.02748^{\star \star}$ \\
\hline $\mathrm{HH}$ & $-0.03338^{*}$ & $-0.0083^{*}$ & $-0.0030^{\star \star}$ & $-0.0109^{* *}$ & $-0.1467^{* \star}$ & $-0.0345^{\star \star}$ & $-0.02847^{\star \star}$ & $-0.031547^{\star *}$ \\
\hline $\mathrm{mpa}$ & & $-0.002014^{\wedge}$ & & & & & & \\
\hline
\end{tabular}

La Tabla presenta en el Panel A el coeficiente de las primas económicas y su nivel de significatividad (separando período completo y subperíodos de crisis del petróleo y financiera, y de expansión económica), para el modelo MRE, separando en A.1: la agrupación por sector y A.2: para tamaño-BM. De forma análoga se facilitan el Panel $B$, para el modelo $F F(m)$.

Nivel de significatividad de los contrastes: $10 \%\left({ }^{\wedge}\right), 5 \%\left({ }^{*}\right)$ y $1 \%\left(^{* *}\right)$.

Fuente: Elaboración propia.

En resumen, el signo que presenta el crecimiento del PIB, desvela que los rendimientos de las carteras responden en sentido contrario a las perspectivas que tienen los inversores sobre el crecimiento económico en todo el período considerado (1993-2011). Estos primeros resultados señalan la importancia del citado factor el cual se agudiza en el período de crisis financiera-08 (20082011). Ello es indicativo de que en épocas de crisis el inversor debe prestar especial atención al crecimiento de la economía (medido por el PIB) en el contexto del mercado de capitales español.

\subsection{Los factores de Fama y French y el efecto momentum en la valoración de activos}

De los resultados presentados en la Tabla 2: Panel A.2 y B.2 y la Figura 1 se pueden extraer las siguientes conclusiones sobre la estimación del modelo $\mathrm{FF}(\mathrm{m})$ (véase ecuación [5]). En términos generales, el modelo $\mathrm{FF}(\mathrm{m})$ proporciona una representación razonable de los datos superior a la proporciona por el modelo CAPM y por lo tanto que el modelo MRE (véase en la Figura 1 la menor anchura de las bandas de confianza), pero peor que la que se deriva del modelo FF.

Cabe señalar que no podemos rechazar la hipótesis de insesgadez de los errores para ambas agrupaciones. Se rechazan las hipótesis conjuntas de riesgos instrumentales nulos y de que todos los riesgos sean iguales entre sí e iguales a cero, respectivamente. Estos resultados subrayan el comportamiento condicional de nuestro modelo y por tanto el acierto al elegir una metodología de estimación no estática. 
En relación a los factores de riesgo asociados al tamaño (SMB) y el ratio book-to-market (HML), las primas asociadas a cada uno de estos riesgos son significativas de forma conjunta. Por otra parte, el efecto momentum a 3, 6, 9 y 12 meses (MOM_k, $k=3,6,9,12$ ) es significativo individual y conjunta. Son significativamente negativos los coeficientes del efecto a 3, 6 y 12 meses (esto es, un efecto contrarian) y significativamente positivo el del efecto a 9 meses (esto es, un efecto momentum).

Los resultados de la Tabla 3: Panel B establecen la importancia de los factores de riesgo asociados al tamaño y ratio book-to-market y confirman (recuérdese que el modelo $\mathrm{FF}(\mathrm{m})$ es peor que el modelo $\mathrm{FF}$ ) el escaso impacto económico del efecto momentum que, posiblemente, se debe a la práctica desaparición del efecto momentum en el mercado de capitales español a partir de la década de los noventa (véase Forner y Marhuenda, 2006). Notemos también que las primas económicas asociadas a los factores tamaño y ratio book-to-market de forma conjunta y las asociadas al factor réplica del PIB son muy similares en signo y magnitud para las dos agrupaciones de carteras, y que el mayor impacto se debe al factor tamaño no produciéndose compensación entre ambos factores.

El impacto económico del factor de negociación momentum es bastante escaso. Para la agrupación por sector resulta ser positivo pero no significativo, y para la agrupación por tamaño-BM es negativo con un nivel de significatividad y valor, muy reducidos.

Con estos resultados se vislumbra que una valoración basada en un modelo que solo considerase el riesgo de mercado implicaría una valoración de los activos financieros españoles inadecuada por el impacto económico que implican los factores de Fama y French (1993). Se observa que el efecto es mucho mayor para la agrupación por tamaño-BM ya que los porcentajes de sobrevaloración llegan a ser del orden del $15.78 \%$, en el peor de los casos. De forma general, estos efectos se agudizan mucho más para la etapa de crisis financiera-08, coincidiendo así con los resultados obtenidos para el modelo MRE.

En cuanto a los mejores modelos, los resultados de la Tabla 4 permiten aseverar que el modelo que mejor explica los rendimientos de las carteras tanto sectoriales (véase Panel A) como agrupadas por tamaño-BM (véase Panel B), de entre los considerados (MRE, FF, FF(m) y CAPM) es el modelo FF, el cual presenta un menor error de estimación, siguiéndole muy de cerca el modelo $\mathrm{FF}(\mathrm{m})$.

Resumiendo, los factores tamaño y ratio book-to-market siguen siendo factores importantes y relevantes para la correcta valoración de activos financieros españoles, ya que el mercado paga primas por los riesgos asociados a estos factores, y el impacto económico de los mismos es significativo para todas las carteras consideradas. El efecto momentum ofrece una relevancia más bien escasa, entra como factor de negociación (y no de riesgo) en la ecuación de valo- 
ración y, aunque presenta coeficientes significativos, su impacto económico es muy reducido. Finalmente, y considerando los errores de estimación de los modelos, el modelo FF es el que mejor describe la variación de los rendimientos de los activos financieros cotizados en la bolsa española.

Tabla 4

Estimación de los mejores modelos de valoración

Panel A: Mejores modelos para la cartera Sector

\begin{tabular}{|c|c|c|c|c|c|c|c|c|c|}
\hline & $\gamma_{0}$ & $\gamma^{\mathrm{m}}$ & $\gamma^{\text {rapib }}$ & $\gamma^{\mathrm{smb}}$ & $\gamma^{\mathrm{hml}}$ & $\gamma^{\text {m-div }}$ & $\gamma^{\text {rapib-div }}$ & $\gamma^{\text {smb-div }}$ & $\gamma^{\text {hmlddiv }}$ \\
\hline$\overline{\mathrm{FF}}$ & $0.02781^{\star \star}$ & $-0.007247^{\wedge}$ & & $0.034781^{\star \star}$ & $-0.03415^{\star \star}$ & -0.002147 & & $-0.02174^{\star \star}$ & $0.01089^{\star \star}$ \\
\hline \multirow[t]{2}{*}{ CAPM } & $0.02187^{* \star}$ & 0.006478 & & & & $-0.00641^{*}$ & & & \\
\hline & $\gamma^{m \cdot \operatorname{tem} m}$ & $\gamma^{\text {rapib-tem }}$ & $\gamma^{\text {smb-term }}$ & $\gamma^{\mathrm{hml}+\mathrm{tem}}$ & $\gamma^{\text {div }}$ & $\gamma^{\text {tem }}$ & Error estim. & & \\
\hline $\mathrm{FF}$ & $-0.0000018^{* \star}$ & & 0.00000217 & $-0.000008^{* *}$ & $-0.00478^{\star \star}$ & -0.0000127 & 0.087401 & & \\
\hline CAPM & $0.000003^{* *}$ & & & & $-0.00148^{\star \star}$ & $-0.000065^{*}$ & 0.214741 & & \\
\hline MRE & & & & & & & 0.354742 & & \\
\hline $\mathrm{FF}(\mathrm{m})$ & & & & & & & 0.187487 & & \\
\hline \multicolumn{10}{|c|}{ Panel B Mejores modelos para la cartera Tamaño-BM } \\
\hline & $\gamma_{0}$ & $\gamma^{m}$ & $\gamma^{\text {rspib }}$ & $\gamma^{\mathrm{smb}}$ & $\gamma^{\mathrm{hml}}$ & $\gamma^{\text {mddiv }}$ & $\gamma^{\text {rapib-div }}$ & $\gamma^{\text {smb-div }}$ & $\gamma^{\text {hmldiv }}$ \\
\hline $\mathrm{FF}$ & $0.01784^{* \star}$ & $-0.02178^{* *}$ & & -0.001974 & 0.004124 & -0.0000587 & & $-0.00978^{* *}$ & $0.00312^{\star *}$ \\
\hline \multirow[t]{2}{*}{ CAPM } & $0.01678^{\star \star}$ & 0.00208 & & & & -0.003145 & & & \\
\hline & $\gamma^{\mathrm{m} \text { them }}$ & $\gamma^{\text {rtsib-tem }}$ & $\gamma^{\text {smb-term }}$ & $\gamma^{\text {hlmlitem }}$ & $\gamma^{\text {div }}$ & $\gamma^{\text {tem }}$ & Error estim. & & \\
\hline$\overline{F F}$ & $-0.0000061^{* \star}$ & & $0.0000062^{* \star}$ & $0.0000021^{*}$ & -0.000461 & $-0.00009^{\star \star}$ & 0.301478 & & \\
\hline CAPM & -0.00000039 & & & & $0.00197^{\star \star}$ & $0.000031^{* *}$ & 1.478411 & & \\
\hline MRE & & & & & & & 1.409978 & & \\
\hline $\mathrm{FF}(\mathrm{m})$ & & & & & & & 0.381146 & & \\
\hline
\end{tabular}

La Tabla presenta, para el período completo, en los Paneles $A$ y B los resultados de la estimación de los modelos FF y CAMP para las carteras sectoriales y tamaño-BM, respectivamente. Los estadísticos proporcionados son: primas al riesgo estimadas, su nivel de significatividad y el error de estimación del modelo. Los datos de los restantes modelos considerados, esto es los modelos $F F(m)$ y MRE, se han facilitado en la Tabla 2 y siven, a su vez, de comparativa entre los presentes para establecer los mejores modelos.

Nivel de significatividad de los contrastes: $10 \%\left(^{\wedge}\right), 5 \%\left(^{*}\right)$ y $1 \%\left(^{* *}\right)$.

Fuente: Elaboración propia.

En resumen, para el período completo (1993-2011) la capacidad explicativa del modelo de Fama y French (1993) para el mercado de capitales español, sigue siendo muy relevante. A diferencia de otros trabajos, nuestros factores momentum participan activamente en la formación de los precios de los activos cotizados, siendo individualmente significativos en todos los casos. Una posible causa de este efecto radica en que se han incorporado en los modelos como factores de negociación y no como factores de riesgo. Independientemente de lo dicho, el modelo que mejor explica la variación de los rendimientos de los activos financieros españoles es el "clásico" modelo de FF, ya que su error estándar es el más reducido. Le sigue muy de cerca el modelo FF(m).

\subsection{Capacidad explicativa racional económica de los factores de Fama y French y efecto momentum a través del factor de riesgo réplica del PIB}

Se ha verificado que el modelo $\mathrm{FF}(\mathrm{m})$ proporciona una buena representación de los rendimientos de los activos españoles que es superior a la proporcionada 
por los modelo CAPM y MRE; en este apartado se aborda el estudio de la naturaleza racional de los efectos tamaño, ratio book-to-market y momentum analizando, dentro del contexto de valoración, la relación entre estos efectos y el factor de riesgo réplica del PIB.

Para hacer este estudio se han analizado las relaciones entre estos factores mediante regresiones múltiples con corrección de errores de Newey y West (1987); y se ha estimado el modelo ${ }^{15} \mathrm{FF}(\mathrm{m})$ aumentado añadiendo el factor réplica del PIB y así comprobar la contribución diferencial de este parámetro sobre los clásicos factores de Fama y French (1993). Para este modelo se calculan los contrastes de significatividad conjunta de las primas asociadas al factor tamaño, el factor ratio book-to-market, los factores tamaño y ratio book-tomarket conjuntamente, el factor réplica del PIB y efecto conjunto momentum, y las primas económicas asociadas a estos factores e impacto económico del efecto momentum para las dos agrupaciones. La Tabla 5 recoge los resultados de las regresiones (Panel A) y de los resultados descritos para el modelo $\mathrm{FF}(\mathrm{m})$ aumentado (Panel B: FF(r $\Delta$ pib)).

A pesar de la similitud observada, en signo y magnitud, entre las primas económicas asociadas a los factores tamaño y ratio book-to-market del modelo $\mathrm{FF}(\mathrm{m})$ y las asociadas al factor réplica del PIB del modelo MRE para las dos agrupaciones de carteras, y contrariamente a las evidencias empíricas de Vassalou (2003), los resultados de la Tabla 5: Panel B no son muy favorables a una interpretación de los factores tamaño y ratio book-to-market como riesgos asociados al factor réplica.

Por consiguiente, en el modelo $\mathrm{FF}(\mathrm{m})$ aumentado con el factor réplica la prima asociada a los factores tamaño y ratio book-to-market se mantiene significativa. Las primas económicas asociadas a los factores tamaño y ratio bookto-market mantienen niveles de significatividad similares e incluso superiores a los del modelo $\mathrm{FF}(\mathrm{m})$ y las primas económicas asociadas al factor réplica se hacen menos significativas para las dos agrupaciones de carteras. El impacto del efecto momentum es similar para el modelo $\mathrm{FF}(\mathrm{m})$ y este modelo $\mathrm{FF}(\mathrm{m})$ aumentado $(\mathrm{FF}(\mathrm{r} \Delta \mathrm{pib}))$. Esto es, el impacto económico del factor réplica sobre los rendimientos de los activos españoles es embebido, en gran medida, por los factores tamaño, ratio book-to-market y efecto momentum. El ajuste de la regresión $\left(\mathrm{R}^{2}\right.$ ajustado $\left.=47.53 \%\right)$ del factor réplica sobre los factores mercado, tamaño, ratio book-to-market y efectos momentum (véase Panel A.2) confirma esta relación inversa.

${ }^{15}$ En este apartado se muestran únicamente los resultados estadísticos para el período completo (1993-2011) ya que los resultados que se obtenían eran muy similares para los subperíodos y por tanto, dado que no habían aportaciones relevantes diferenciadoras, se han omitido. 


\section{Tabla 5}

Capacidad explicativa de los factores y contrastes de significatividad de las primas económicas, para el modelo $\mathrm{FF}(\mathrm{m})$ aumentado con el factor réplica del PIB ( $F F(r \Delta \mathrm{pib}))$

Panel A: Capacidad explicativa de los factores

Panel A.1: Capacidad del factor RAPIB, para explicar los efectos tamaño, ratio book-to-market y momentum

\begin{tabular}{|c|c|c|c|c|c|c|}
\hline & Constante & EXM & $R \Delta P I B$ & $\operatorname{div}(-1)$ & term $(-1)$ & $\mathrm{R}^{2}$ Ajustado \\
\hline SMB & 0.012811 & $-0.213601^{*}$ & 0.041803 & -0.059279 & -4.201119 & 0.068741 \\
\hline HML & $0.021086^{\star *}$ & -0.062143 & $1.41132^{* \star}$ & $-0.111445^{\star *}$ & $-15.24433^{\star \star}$ & 0.229741 \\
\hline MOM_6 & -0.0000286 & -0.081236 & 0.21003 & 0.010667 & -3.730793 & -0.000102 \\
\hline MOM_9 & 0.00706 & 0.009482 & 0.100112 & -0.001841 & $5.612783^{\wedge}$ & 0.080247 \\
\hline MOM_12 & 0.002248 & -0.084008 & 0.118852 & 0.015423 & 3.417826 & 0.091462 \\
\hline
\end{tabular}

Panel A.2: Capacidad de los efectos tamaño, ratio book-to-market y momentum para explicar el factor RAPIB

\begin{tabular}{|c|c|c|c|c|c|c|c|c|}
\hline & Constante & EXM & SMB & HML & MOM_3 & MOM_6 & MOM_9 & MOM_12 \\
\hline $\mathrm{R} \triangle \mathrm{PIB}$ & -0.00409 & -0.013009 & 0.022041 & $0.145615^{\star \star}$ & -0.016603 & -0.019108 & -0.041008 & $0.084178^{* *}$ \\
\hline & $\operatorname{div}(-1)$ & term(-1) & $\mathbf{R}^{2}$ Ajustado & & & & & \\
\hline
\end{tabular}

Panel B: Modelo FF(m) aumentado con el factor réplica del PIB (FF(r $\Delta p$ pib))

Panel B.1: Carteras por Sector

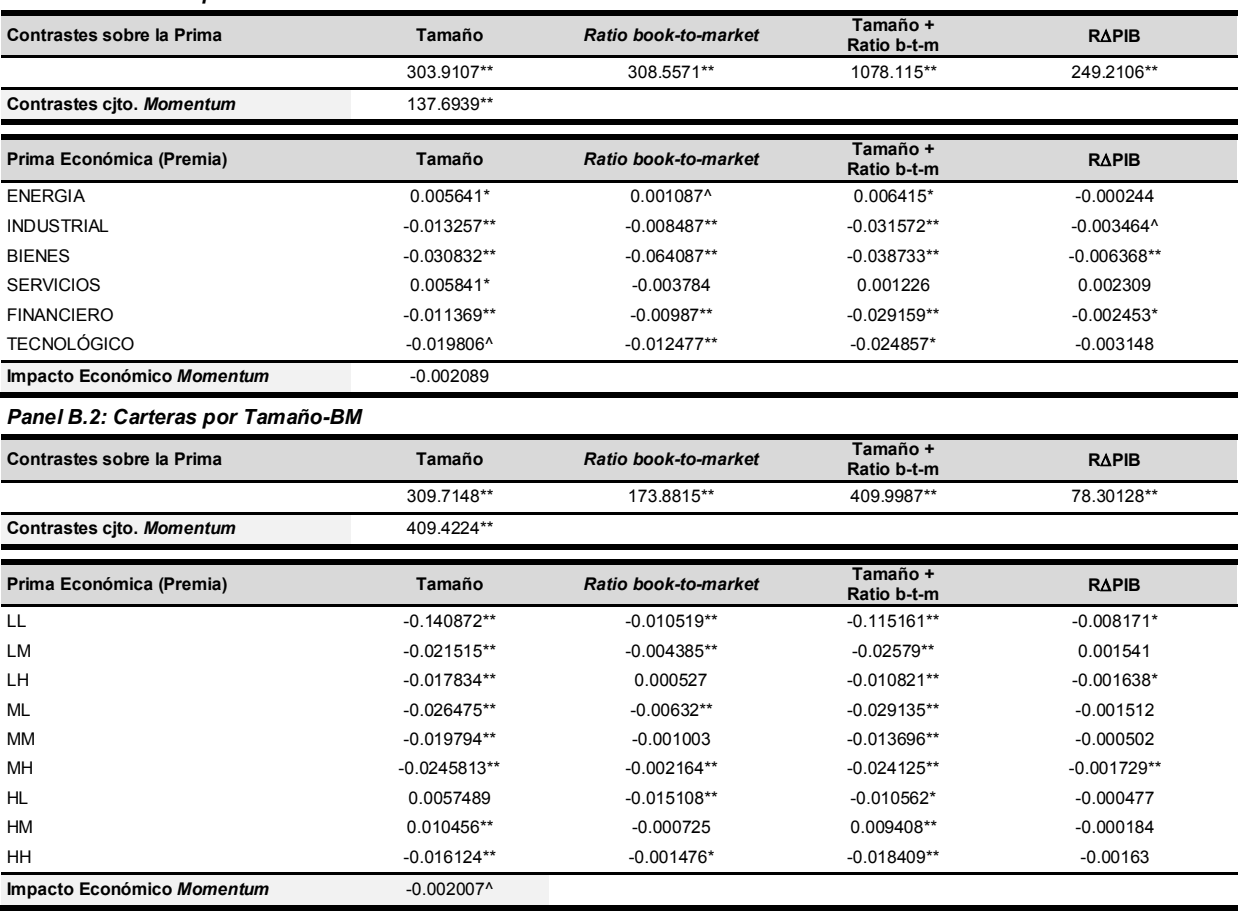

En el panel A de la Tabla, aparecen los resultados estadísticos de los contrastes de significatividad individual de Wald para los modelos de valoración, siendo el factor fila la variable explicada y los dispuestos por columnas, las variables explicativas. Se detallan adicionalmente los resultados de los contrastes de significatividad individual de Wald para el modelo $F F(m)$ agregando el factor réplica del PIB (Panel B). Los contrastes de Wald sobre la prima, en los Paneles B.1 y B.2, representan los coeficientes de regresión y su significatividad para el modelo $F F(m)$ aumentado con el factor RAPIB. Seguidamente se facilitan los impactos económicos para todos los modelos.

Nivel de significatividad de los contrastes: $10 \%\left(^{\wedge}\right), 5 \%\left(^{*}\right)$ y $1 \%\left(^{* *}\right)$.

Fuente: Elaboración propia. 
En conclusión, el factor réplica del crecimiento futuro del PIB aporta escasa información incremental, en materia de valoración, respecto a la proporcionada por los "clásicos" factores de riesgo del modelo de Fama y French (1993).

\section{CONCLUSIONES}

Con este trabajo nos planteamos dos objetivos fundamentales: (i) cuantificar la contribución de los factores tamaño, ratio book-to-market y momentum, en términos de primas y del impacto económico de las mismas para explicar los rendimientos de sección cruzada de activos españoles, separando la muestra completa (1993-2011) en: fase de crisis económica-93 (1993-1997), fase de crecimiento económico (1998-2007), y fase de crisis financiera-08 (2008-2011); y (ii) analizar si estos factores admiten una explicación racional económica, como factores asociados a la información del mercado sobre el crecimiento económico futuro y por tanto, medidores de los riesgos económicos futuros. Los resultados obtenidos se pueden resumir en tres aspectos generales:

- El modelo con factores de riesgo de mercado y réplica del PIB proporciona una representación de los rendimientos de los activos españoles asimilable a la proporcionada por el modelo de tres factores de Fama y French (1993) con factor (de negociación) momentum. Efectivamente, los impactos económicos asociados al factor réplica del PIB y a los factores tamaño y ratio book-to-market son de una magnitud muy similar para las dos agrupaciones de carteras consideradas. Estos resultados ponen de manifiesto que el crecimiento futuro del PIB es un factor relevante en el proceso de formación de los precios para los activos financieros cotizados en la bolsa española; relevancia que adquiere mayor notoriedad a partir de la crisis financiera iniciada en 2008.

- El modelo de tres factores de Fama y French (1993) es el modelo que mejor describe la variación de los rendimientos de activos españoles en el periodo estudiado. Verificamos que el impacto económico de los factores momentum a 3, 6, 9 y 12 no es significativo para ninguna de las dos agrupaciones. Esto nos indica nuevamente que omitir estos factores (tamaño y ratio book-to-market) en el proceso de valoración implicaría que la mayoría de las carteras, para cualquier inversor, estarían infra/sobrevaloradas por el impacto de las primas económicas.

- Los resultados sobre el impacto económico de los factores revelan una interpretación débil de los factores tamaño y ratio book-to-market como riesgos asociados a la réplica del PIB. En efecto, al aumentar el modelo de Fama y French con los factores (de negociación) momentum más el factor réplica del PIB; las primas económicas asociadas a los factores tamaño y ratio book-to-market mantienen niveles de significatividad similares e incluso superiores a las del modelo de Fama y French extendido sólo 
con factores (de negociación) momentum. Asimismo, las primas económicas asociadas al factor réplica del PIB se hacen menos significativas para las dos agrupaciones de carteras. Por tanto, la capacidad explicativa del factor réplica del PIB cuando se integra con los "clásicos" factores de Fama y French, es prácticamente embebida dejando patente su escasa aportación incremental.

En conclusión, llevar a cabo una correcta valoración de los activos financieros en cualquier mercado de capitales es crucial, y para ello es fundamental identificar los riesgos que tienen una participación activa en la formación de los precios, por las severas implicaciones que ello supone en la correcta valoración de los activos cotizados. Los resultados empíricos del presente trabajo desvelan que siguen teniendo un peso relevante, en este proceso de valoración, los "clásicos" riesgos asociados al tamaño de las empresas y al ratio book-to-market, caso contrario para el efecto momentum. Aunque se encuentra una interpretación económica débil de estos dos factores a partir de la cartera réplica que recoge las noticias del mercado sobre el crecimiento futuro del PIB. A su vez, se ha demostrado que la información contenida en el factor réplica del PIB también es importante, cuya justificación se halla en la significatividad en términos de prima al riesgo e impactos económicos. Finalmente destacar que, omitir estos riesgos supone, una incorrecta valoración de los activos financieros de la bolsa española, que sería mucho más aguda en las fases de crisis (económica-93 y especialmente financiera-08), donde los impactos económicos se elevan.

\section{REFERENCIAS BIBLIOGRÁFICAS}

BLACK, F. (1972). "Capital Market Equilibrium with Restricted Borrowing" en Journal of Business, 45, pp. 444-455.

CARHART, M. M. (1997). "On Persistence in Mutual Fund Performance" en Journal of Finance, 52(1), pp. 57-82.

CHEN, N.F. (1983). "Some Empirical Tests of the Theory of Arbitrage Pricing" en Journal of Finance, 38, pp. 1393-1414.

COCHRANE, J.H. (1996). "A Cross-Sectional Test of an Investment based Asset Pricing Models" en Journal of Political Economy, 104, pp. 572-621.

CONRAD, J., M.N. GULTEKIN, y KAUL, G. (1991). "Asymmetric Predictability of Condicional Variances" en Review of Financial Studies, 4, pp. 597-622.

DE SANTIS, G. y GERARD, B. (1997). "International Asset Pricing and Portfolio Diversification with Time-Varying Risk" en Journal of Finance, 52, pp. 1881-1912.

DE SANTIS, G., B. GERARD, y HILLION, P. (2003). "The Relevance of Currency Risk in the EMU" en Journal of Economics and Business, 55, pp. 427-462.

DUARTE, A., VENETIS, I. y PAYÁ, I. (2004). "Curva de rendimientos y crecimiento de la producción real en la UEM: eficiencia y estabilidad predictiva” en Estudios de Eco- 
nomía Aplicada, 22(1), pp. 1-21.

DUMAS, B. y SOLNIK, B. (1995). "The World Price of Foreign Exchange risk" en Journal of Finance, 50(2), pp. 445-479.

EEAG (2011). The EEAG Report on the European Economy, CESifo, Munich, 2010. Documento de Trabajo. http://www.cesifo-group.de/portal/pls/portal/docs/1/1201535.pdf [Último acceso: Octubre de 2013].

FAMA, E.F. y FRENCH, K.R. (1988). "Dividend Yields and Expected Stock Returns" en Journal of Financial Economics, 22, pp. 3-27.

FAMA, E.F. y FRENCH, K.R. (1989). "Business Conditions and Expected Returns on Stocks and Bonds" en Journal of Financial Economics, 25, pp. 23-49.

FAMA, E.F. y FRENCH, K.R. (1993). "Common Risk Factors in the Returns on Stoks and Bonds" en Journal of Financial Economics, 33(1), pp. 3-56.

FAMA, E.F. y FRENCH, K.R. (1995)." Size and Book-to-Market Factors in Earnings and Returns" en Journal of Finance, 50, pp. 131-155.

FAMA, E.F. y FRENCH, K.R. (1996). "Multifactor Explanations for Asset Pricing Anomalies" en Journal of Finance, 51, pp. 55-84.

FAMA, E.F. y MACBETH, J.D. (1973). "Risk, Return, and Equilibrium: Empirical Tests" en Journal of Political Economy, 81, pp. 607-636.

FERSON, W.E. y HARVEY, C.R. (1991). "The Variation of Economic Risk Premiums” en Journal of Political Economics, 99, pp. 385-415.

FERSON, W.E. y HARVEY, C.R. (1999). "Conditioning Variables and Cross-Section of Stock Returns" en Journal of Finance, 54, pp. 1325-1360.

FONT, B. y GRAU, A.J. (2007). "Los Factores Tamaño, Book-to Market y Momentum en el Mercado de Capitales Español: Explicaciones Racionales en la Formación del Precio" en Revista Española de Financiación y Contabilidad, 36, pp. 509-536.

FONT, B. y GRAU, A.J. (2009). “¿Cómo se valoran las acciones españolas: en el mercado de capitales doméstico o en un mercado europeo?" en Moneda y Crédito, 229, pp. 91-155.

FORNER, C. y MARHUENDA, J. (2001). “¿Existe en el mercado español un efecto sobre-reacción?” en Revista española de financiación y contabilidad, 107, pp. 39-66.

FORNER, C. y MARHUENDA, J. (2003). "Contrarian and Momentum Strategies in the Spanish Stock Market” en European Financial Management, 9, pp. 67-88.

FORNER, C. y MARHUENDA, J. (2004). El efecto sobre-reacción en el mercado español" en Moneda y Crédito, 219, pp. 177-182.

FORNER, C. y MARHUENDA, J. (2006). "Análisis del Origen de los Beneficios del Momentum en el Mercado de Valores Español” en Investigaciones Económicas, 30, pp. 401-439.

GIBBONS, M.R. (1982). "Multivariate Test of Financial Models. A New Approach" en Journal of Financial Economics, 10, pp. 3-27.

JAGANNATHAN, R. y WANG, Z. (1996). "The conditional CAPM and the cross-section of expected returns" en Journal of Finance, 51, pp. 3-53.

JEGADEESH, N. y TITMAN, S. (1993). "Returns to Buying Winners and Selling Losers: Implications for Stock Market Efficiency" en Journal of Finance, 48, pp. 65-91.

LAMONT, A. (2001). "Economic Tracking Portfolios" en Journal of Econome-trics, 105, pp. 161-184. 
LIEW, J. y VASSALOU, M. (2000). "Can Book-to-Market, Size and Momentum be Risk Factors that Predict Economic Growth?" en Journal of Financial E-conomics, 57, pp. 221-245.

MERTON, R. (1973). "An Intemporal Capital Asset Pricing Model” en Econometrica, 41, pp. 867-887.

MIRALLES, J.L. y MIRALLES, M.M. (2003). "Actividad Negociadora y Esperanza de Rentabilidad en la Bolsa de Valores Española" en Revista Economía Financiera, 1, pp. 15-36.

MIRALLES, J.L., MIRALLES, M.M. y MIRALLES, J.L. (2007). "Análisis media-semivarianza: una aplicación a las primas de riesgo en el mercado de valores español" en Estudios de Economía Aplicada, 25(1), pp.199-214.

MOSKOWITZ, T.J. y GRINBLATT, M. (1999). "Do Industries Explain Momentum?" en Journal of Finance, 54(4), pp. 1.249-1.290.

MUGA, L. y SANTAMARÍA, R. (2006). "Momentum: características y estabilidad temporal. Resultados para la bolsa española" en Revista Española de Financiación y Contabilidad, 130, pp. 597-620.

MUGA, L. y SANTAMARÍA, R. (2007). "Riesgo asimétrico y estrategias de momentum en el mercado de valores español" en Investigaciones Económicas, 31, pp. 323-340.

NEWEY, W. y WEST, K. (1987). "A Simple Positive Semi-Definite, Heteroskedasticity and Autocorrelation Consistent Covariance Matrix" en Econometrica, 55, pp. 703708.

NIETO, B. (2002). "La valoración intertemporal de activos: un análisis empírico para el mercado español de valores" en Investigaciones Económicas, 26, pp. 497-524.

NIETO, B. (2004). "Evaluating Multi-Beta Pricing Models: An Empirical Analysis with Spanish Market Data” en Revista de Economía Financiera, 2, pp. 80-108.

NIETO, B. y RODRÍGUEZ, R. (2002): The compsumption-wealth and book-to-market ratios in a dynamic asset context. Documento de trabajo. Instituto Valenciano de Investigaciones Económicas. http://www.ivie.es/downloads/docs/wpasec/wpasec2002-24.pdf. [Último acceso: Octubre de 2013].

NIETO, B. y RODRÍGUEZ, R. (2005). "Modelos de Valoración de Activos Condicionales: Un Panorama Comparativo" en Investigaciones Económicas, 29, pp. 33-71.

NIETO, B. y RUBIO, G. (2002). "El modelo de valoración con cartera de mercado: una nueva especificación del coeficiente beta" en Revista Española de Financiación y Contabilidad, 113, pp. 697-723.

RUBIO, G. (1991). "Formación de Precios en el Mercado Bursátil: Teoría y Evidencia Empírica" en Cuadernos Económicos de ICE, 49, pp. 157-186.

SHANKEN, J. (1992). "On the Estimation of Beta-Pricing Models" en Review of Financial Studies, 5, pp. 1-34.

VASSALOU, M. (2003). "News related to Future GDP Growth as a Risk Factor in Equity Returns"en Journal of Financial Economics, 68, pp. 47-73. 